\title{
GLOBAL EXISTENCE AND SCATTERING FOR A CLASS OF NONLINEAR FOURTH-ORDER SCHRÖDINGER EQUATION BELOW THE ENERGY SPACE
}

\author{
VAN DUONG DINH
}

\begin{abstract}
In this paper, we consider a class of nonlinear fourth-order Schrödinger equation, namely

$$
\left\{\begin{aligned}
i \partial_{t} u+\Delta^{2} u & =-|u|^{\nu-1} u, \quad 1+\frac{8}{d}<\nu<1+\frac{8}{d-4}, \\
u(0) & =u_{0} \in H^{\gamma}\left(\mathbb{R}^{d}\right), \quad 5 \leq d \leq 11 .
\end{aligned}\right.
$$

Using the $I$-method combined with the interaction Morawetz inequality, we establish the global well-posedness and scattering in $H^{\gamma}\left(\mathbb{R}^{d}\right)$ with $\gamma(d, \nu)<\gamma<2$ for some value $\gamma(d, \nu)>0$.
\end{abstract}

\section{INTRODUCTION}

Consider the following nonlinear fourth-order Schrödinger equation

$$
\left\{\begin{aligned}
i \partial_{t} u(t, x)+\Delta^{2} u(t, x) & =-\left(|u|^{\nu-1} u\right)(t, x), \quad t \in \mathbb{R}, x \in \mathbb{R}^{d} \\
u(0, x) & =u_{0}(x) \in H^{\gamma}\left(\mathbb{R}^{d}\right),
\end{aligned}\right.
$$

where $u(t, x)$ is a complex valued function in $\mathbb{R} \times \mathbb{R}^{d}, d \geq 5$. The nonlinear exponent $\nu$ is assumed to be mass-supercritical, i.e $\nu>1+\frac{8}{d}$ and energy-subcritical, i.e. $\nu<1+\frac{8}{d-4}$. The regularity exponent $\gamma$ is assumed to satisfy $0<\gamma<2$.

The fourth-order Schrödinger equation was introduced by Karpman [Kar96] and KarpmanShagalov [KS00] to take into account the role of small fourth-order dispersion terms in the propagation of intense laser beams in a bulk medium with Kerr nonlinearity. Such a fourth-order Schrödinger equation is of the form

$$
i \partial_{t} u+\Delta^{2} u+\varepsilon \Delta u+\mu|u|^{\nu-1} u=0, \quad u(0)=u_{0},
$$

where $\varepsilon \in\{0, \pm 1\}, \mu \in\{ \pm 1\}$ and $\nu>1$. We note that (NL4S) is a special case of (1.1) by taking $\varepsilon=0$ and $\mu=1$. The nonlinear fourth-order Schrödinger equation (1.1) has attracted a lot of interest in a past decay. The sharp dispersive estimates for the linear part of (1.1) were established in [BKS00]. The local well-posedness and the global well-posedness for (1.1) has been widely studied in [Din1, Din2, Din3, Din4, Guo10, GC06, HHW06, HHW07, HJ05, MXZ09, MXZ11, MWZ15, MZ07, Pau1, Pau2, PS10] and references therein.

The (NL4S) enjoys a natural scaling invariance, that is if we set for $\lambda>0$

$$
u_{\lambda}(t, x):=\lambda^{-\frac{4}{\nu-1}} u\left(\lambda^{-4} t, \lambda^{-1} x\right),
$$

then for $T \in(0,+\infty]$,

$$
u \text { solves (NL4S) on }(-T, T) \Longleftrightarrow u_{\lambda} \text { solves (NL4S) on }\left(-\lambda^{4} T, \lambda^{4} T\right) \text {. }
$$

2010 Mathematics Subject Classification. 35G20, 35G25, 35Q55.

Key words and phrases. Nonlinear fourth-order Schrödinger equation; Global well-posedness; Scattering; Almost conservation law; Morawetz inequality. 
We define the critical regularity exponent for (NL4S) by

$$
\gamma_{\mathrm{c}}:=\frac{d}{2}-\frac{4}{\nu-1}
$$

The (NL4S) is known (see [Din1] or [Din2]) to be locally well-posed in $H^{\gamma}\left(\mathbb{R}^{d}\right)$ with $\gamma \geq \max \left\{0, \gamma_{c}\right\}$ satisfying for $\nu$ is not an odd integer,

$$
\lceil\gamma\rceil \leq \nu
$$

Here $\lceil\gamma\rceil$ is the smallest integer greater than or equal to $\gamma$. This condition ensures the nonlinearity to have enough regularity. In the sub-critical regime, i.e. $\gamma>\gamma_{c}$, the time of existence depends only on the $H^{\gamma}$-norm of initial data. Moreover, the local solution enjoys mass conservation, i.e.

$$
M(u(t)):=\|u(t)\|_{L^{2}\left(\mathbb{R}^{d}\right)}^{2}=\left\|u_{0}\right\|_{L^{2}\left(\mathbb{R}^{d}\right)}^{2},
$$

and $H^{2}$-solution has conserved energy, i.e.

$$
E(u(t)):=\int_{\mathbb{R}^{d}} \frac{1}{2}|\Delta u(t, x)|^{2}+\frac{1}{\nu+1}|u(t, x)|^{\nu+1} d x=E\left(u_{0}\right) .
$$

The persistence of regularity (see [Din2]) combined with the conservations of mass and energy yield the global well-posedness for (NL4S) in $H^{\gamma}\left(\mathbb{R}^{d}\right)$ with $\gamma \geq 2$ satisfying for $\nu$ is not an odd integer, (1.4). In the critical regime, i.e. $\gamma=\gamma_{c}$, one also has (see [Din1] or [Din2]) the local well-posedness for (NL4S) but the time of existence depends not only on the $H^{\gamma}$-norm of initial data but also on its profile. Moreover, for small initial data, the (NL4S) is globally well-posed, and the solution is scattering.

The main goal of this paper is to show the global well-posedness and scattering for the nonlinear fourth-order Schrödinger equation (NL4S) below the energy space. Our arguments are based on the combination of the $I$-method and the interaction Morawetz inequality which are similar to those of [VZ09]. However, there are some difficulties due to the high-order dispersion term $\Delta^{2} u$. Moreover, in order to successfully establish the almost conservation law, we need the nonlinearity to have at least two orders of derivatives. This leads to the restriction in spatial space of dimensions $5 \leq d \leq 11$.

Before stating our main result, let us recall some known results concerning the global existence below the energy space for the nonlinear fourth-order Schrödinger equation. To our knowledge, Guo in [Guo10] gave a first answer to this problem. In [Guo10], the author considered (1.1) with $\nu-1=2 m, m \in \mathbb{N}$ satisfying $4<m d<4 m+2$, and established the global existence in $H^{\gamma}\left(\mathbb{R}^{d}\right)$ with $1+\frac{m d-9+\sqrt{(4 m-m d+7)^{2}+16}}{4 m}<\gamma<2$. The proof is based on the $I$-method which is a modification of the one invented by $I$-Team [CKSTT02] in the context of nonlinear Schrödinger equation. Later, Miao-Wu-Zhang in [MWZ15] studied the defocusing cubic fourth-order Schrödinger equation, i.e. $\nu=3$ in (NL4S), and proved the global well-posedness and scattering in $H^{\gamma}\left(\mathbb{R}^{d}\right)$ with $\gamma(d)<\gamma<2$ where $\gamma(5)=\frac{16}{11}, \gamma(6)=\frac{16}{9}$ and $\gamma(7)=\frac{45}{23}$. The proof relies on the combination of the $I$-method and a new interaction Morawetz inequality. Recently, in [Din3] the author considered the defocusing cubic higher-order Schrödinger equation including the cubic fourth-order Schrödinger equation, and showed that the (NL4S) with $\nu=3$ is globally well-posed in $H^{\gamma}\left(\mathbb{R}^{4}\right)$ with $\frac{60}{53}<\gamma<2$. The argument makes use of the $I$-method and the bilinear Strichartz estimate. The analysis is carried out in Bourgain spaces $X^{\gamma, b}$ which is similar to those in [CKSTT02]. In the above considerations, the nonlinearity is algebraic, i.e. $\nu$ is an odd integer. This allows to write the commutator between the $I$-operator and the nonlinearity explicitly by means of the Fourier transform, and then carefully control the frequency interactions using multi-linear analysis. When one considers the nonlinear fourth-order Schrödinger equation (NL4S) with $\nu>1$ is not an odd integer, this method does not 
work. We thus rely purely on Strichartz and interaction Morawetz estimates.

Let us now introduce some notations.

$$
\gamma(d, \nu):=\max \left\{\gamma_{1}(d, \nu), \gamma_{2}(d, \nu), \gamma_{3}(d, \nu), \gamma_{4}(d, \nu)\right\}
$$

where

$$
\begin{aligned}
\gamma_{1}(d, \nu) & :=\frac{3}{2}+\frac{\gamma_{\mathrm{c}}}{4}, \\
\gamma_{2}(d, \nu) & :=4-\nu \\
\gamma_{3}(d, \nu) & :=\frac{2}{\nu-1}+\frac{(\nu-2) \gamma_{\mathrm{c}}}{\nu-1}, \\
\gamma_{4}(d, \nu) & :=\min _{\sigma \in\left(0, \sigma_{0}\right]} \gamma(d, \nu, \sigma) .
\end{aligned}
$$

Here $\sigma_{0}$ satisfies

$$
\left\{\begin{aligned}
2 \sigma_{0}(16-(\nu-1)(d+4)) & <(d-5)(d(\nu-1)-8), \\
2 \sigma_{0}(\nu-3) & \leq d-5 \\
\sigma_{0} & \leq \gamma
\end{aligned}\right.
$$

and $\gamma(d, \nu, \sigma)$ is the (large if there are two) root of the equation

$$
\gamma_{\mathrm{c}}(2-\gamma)(d-5+(8-d) \sigma)=\min \left\{\gamma-1-\frac{\gamma_{\mathrm{c}}}{2}, \nu-2,(\nu-2)\left(\gamma-\gamma_{\mathrm{c}}\right)\right\}\left(\gamma-\gamma_{\mathrm{c}}\right) \sigma
$$

The main result of this paper is the following:

Theorem 1.1. Let $5 \leq d \leq 11$. The initial value problem (NL4S) is globally well-posed in $H^{\gamma}\left(\mathbb{R}^{d}\right)$ for any $\gamma(d, \nu)<\gamma<2$, and the global solution $u$ enjoys the following uniform bound

$$
\|u\|_{L^{\infty}\left(\mathbb{R}, H^{\gamma}\left(\mathbb{R}^{d}\right)\right)} \leq C\left(\left\|u_{0}\right\|_{H^{\gamma}\left(\mathbb{R}^{d}\right)}\right) .
$$

Moreover, the solution is scattering, i.e. there exist unique $u_{0}^{ \pm} \in H^{\gamma}\left(\mathbb{R}^{d}\right)$ such that

$$
\lim _{t \rightarrow \pm \infty}\left\|u(t)-e^{i t \Delta^{2}} u_{0}^{ \pm}\right\|_{H^{\gamma}\left(\mathbb{R}^{d}\right)}=0 .
$$

We record in the table below some best known results, and compare them with our ones. As in the table, our results are not as good as the best known results when $\nu$ is an odd integer. But our method allows to treat the non-algebraic nonlinearity.

\begin{tabular}{|c|c|c|c|c|}
\hline$\nu$ & $d$ & $\gamma_{\mathrm{c}}$ & $\gamma(d, \nu)$ (best known results) & $\gamma(d, \nu)$ (our results) \\
\hline \multirow{3}{*}{3} & 5 & $\frac{1}{2}$ & $\frac{16}{11} \approx 1.4545($ see $[\mathrm{MWZ15}])$ & 1.6711 \\
\cline { 2 - 6 } & 6 & 1 & $\frac{16}{9} \approx 1.7777($ see $[\mathrm{MWZ15}])$ & 1.8719 \\
\cline { 2 - 5 } & 7 & $\frac{3}{2}$ & $\frac{45}{23} \approx 1.9565($ see $[\mathrm{MWZ15}])$ & 1.9665 \\
\hline \multirow{2}{*}{4} & 5 & $\frac{7}{6}$ & - & 1.9257 \\
\cline { 2 - 5 } & 6 & $\frac{5}{3}$ & - & 1.9922 \\
\hline
\end{tabular}

TABLE 1. Our results compare with best known results.

The proof of the above result is based on two main ingredients: the $I$-method and the interaction Morawetz inequality, which are similar to those given in [VZ09]. The $I$-method for the fourthorder Schrödinger equation is a modification of the one introduced by I-Team in [CKSTT02]. This method is very useful for treating the nonlinear dispersive equation at low regularity, i.e. below 
energy space. The idea is to replace the non-conserved energy $E(u)$ when $\gamma<2$ by an "almost conserved" variance $E(I u)$ with $I$ a smoothing operator which is the identity at low frequency and behaves like a fractional integral operator of order $2-\gamma$ at high frequency. Since $I u$ is not a solution of (NL4S), we may expect an energy increment. The key is to show that the modified energy $E(I u)$ is an "almost conserved" quantity in the sense that the time derivative of $E(I u)$ decays with respect to a large parameter $N$ (see Section 2 for the definition of $I$ and $N$ ). To do so, we need delicate estimates on the commutator between the $I$-operator and the nonlinearity. When the nonlinearity is algebraic, we can use the Fourier transform to write this commutator explicitly, and then carefully control the frequency interactions. Once the nonlinearity is no longer algebraic, this method fails. In order to treat this case, we take the advantage of Strichartz estimate with a gain of derivatives (2.5). Thanks to this Strichartz estimate, we are able to apply the technique given in [VZ09] to control the commutator. Of course, this technique is not as good as the Fourier transform technique when the nonlinearity is algebraic, but it is more robust and allows us to treat the non-algebraic nonlinearity. The interaction Morawetz inequality for the nonlinear fourth-order Schrödinger equation was first introduced in [Pau2] for $d \geq 7$. Then, it was extended for $d \geq 5$ in [MWZ15]. Using this interaction Morawetz inequality and the interpolation argument together with the Sobolev embedding, we have for any compact interval $J$ and $0<\sigma \leq \gamma$,

$$
\|u\|_{M^{\sigma}(J)}:=\|u\|_{L_{t}^{\frac{d-5+4 \sigma}{\sigma}}\left(J, L_{x} \frac{2(d-5+4 \sigma)}{d-5+2 \sigma}\right)} \lesssim\left(\left\|u_{0}\right\|_{L_{x}^{2}}\|u\|_{L_{t}^{\infty}\left(J, \dot{H}_{x}^{\frac{1}{2}}\right)}\right)^{\frac{2 \sigma}{d-5+4 \sigma}}\|u\|_{L_{t}^{\infty}\left(J, \dot{H}_{x}^{\sigma}\right)}^{\frac{d-5}{d-5+4 \sigma}} .
$$

As a byproduct of the Strichartz estimates and $I$-method, we show the "almost conservation law" for (NL4S), that is if $u \in L^{\infty}\left(J, \mathscr{S}\left(\mathbb{R}^{d}\right)\right)$ is a solution to (NL4S) on a time interval $J=[0, T]$, and satisfies $\left\|I u_{0}\right\|_{H_{x}^{2}} \leq 1$ and if $u$ satisfies in addition the a priori bound $\|u\|_{M^{\sigma}(J)} \leq \mu$ for some small constant $\mu>0$, then

$$
\sup _{t \in[0, T]}\left|E(I u(t))-E\left(I u_{0}\right)\right| \lesssim N^{-(2-\gamma+\delta)},
$$

for some $\delta>0$.

We now give an outline of the proof. Let $u$ be a global in time solution to (NL4S) with initial data $u_{0} \in C_{0}^{\infty}\left(\mathbb{R}^{d}\right)$. Our goal to to show the uniform bounds

$$
\begin{aligned}
\|u\|_{M^{\sigma}(\mathbb{R})} & \leq C\left(\left\|u_{0}\right\|_{H_{x}^{\gamma}}\right), \\
\|u\|_{L_{t}^{\infty}\left(\mathbb{R}, H_{x}^{\gamma}\right)} & \leq C\left(\left\|u_{0}\right\|_{H_{x}^{\gamma}}\right),
\end{aligned}
$$

Thanks to (1.9), the global existence follows immediately by a standard density argument. Since $E\left(I u_{0}\right)$ is not necessarily small, we will use the scaling (1.2) to make $E\left(I u_{\lambda}(0)\right)$ small in order to apply the "almost conservation law". By choosing

$$
\lambda \sim N^{\frac{2-\gamma}{\gamma-\gamma_{\mathrm{c}}}}
$$

and using some harmonic analysis, we can make $E\left(I u_{\lambda}(0)\right) \leq \frac{1}{4}$. We will show that there exists an absolute constant $C$ such that

$$
\left\|u_{\lambda}\right\|_{M^{\sigma}(\mathbb{R})} \leq C \lambda^{\gamma_{\mathrm{c}}+\frac{\sigma(4-d) \gamma_{\mathrm{c}}}{2(d-5+4 \sigma)}}
$$

We then obtain (1.8) by undoing the scaling. In order to prove (1.11), we perform a bootstrap argument. Note that (1.11) is equivalent to

$$
\left\|u_{\lambda}\right\|_{M^{\sigma}([0, t])} \leq C \lambda^{\gamma_{c}+\frac{\sigma(4-d) \gamma_{c}}{2(d-5+4 \sigma)}}, \quad \forall t \in \mathbb{R} .
$$


Assume by contraction, it is not so. Since $\left\|u_{\lambda}\right\|_{M^{\sigma}([0, t])}$ is a continuous function in $t$, there exists $T>0$ so that

$$
\begin{gathered}
\left\|u_{\lambda}\right\|_{M^{\sigma}([0, T])}>C \lambda^{\gamma_{c}+\frac{\sigma(4-d) \gamma_{c}}{2(d-5+4 \sigma)}}, \\
\left\|u_{\lambda}\right\|_{M^{\sigma}([0, T])} \leq 2 C \lambda^{\gamma_{c}+\frac{\sigma(4-d) \gamma_{c}}{2(d-5+4 \sigma)}} .
\end{gathered}
$$

Using (1.13), we can split $[0, T]$ into $L$ subintervals $J_{k}, k=1, \ldots, L$ so that

$$
\left\|u_{\lambda}\right\|_{M^{\sigma}\left(J_{k}\right)} \leq \mu \text {. }
$$

The number $L$ must satisfy

$$
L \sim \lambda^{\frac{\gamma_{c}(d-5+(8-d) \sigma)}{\sigma}} .
$$

We thus can apply the "almost conservation law" to get

$$
\sup _{[0, T]} E\left(I u_{\lambda}(t)\right) \leq E\left(I u_{\lambda}(0)\right)+N^{-(2-\gamma+\delta)} L .
$$

Since $E\left(I u_{\lambda}(0)\right) \leq \frac{1}{4}$, we need

$$
N^{-(2-\gamma+\delta)} L \ll \frac{1}{4}
$$

in order to guarantee $E\left(I u_{\lambda}(t)\right) \leq 1$ for all $t \in[0, T]$. Combining (1.10), (1.14) and (1.15), we get a condition on $\gamma$. Next, by (1.7) and some harmonic analysis, we have

$$
\begin{aligned}
\left\|u_{\lambda}\right\|_{M^{\sigma}([0, T])} \leq C\left(\left\|u_{0}\right\|_{L_{x}^{2}}\right) \lambda^{\gamma_{c}+\frac{\sigma(4-d) \gamma_{c}}{2(d-5+4 \sigma)}} \sup _{[0, T]}\left(\left\|I u_{\lambda}(t)\right\|_{\dot{H}_{x}^{2}}^{\frac{1}{4}}+\left\|I u_{\lambda}(t)\right\|_{\dot{H}_{x}^{2}}^{\frac{1}{2 \gamma}}\right)^{\frac{2 \sigma}{d-5+4 \sigma}} & \\
& \times \sup _{[0, T]}\left(\left\|I u_{\lambda}(t)\right\|_{\dot{H}_{x}^{2}}^{\frac{\sigma}{2}}+\left\|I u_{\lambda}(t)\right\|_{\dot{H}_{x}^{2}}^{\frac{\sigma}{\gamma}}\right)^{\frac{d-5}{d-5+4 \sigma}} .
\end{aligned}
$$

Since $\left\|I u_{\lambda}(t)\right\|_{\dot{H}_{x}^{2}}^{2} \lesssim E\left(I u_{\lambda}(t)\right) \leq 1$ for all $t \in[0, T]$, we get

$$
\left\|u_{\lambda}\right\|_{M^{\sigma}([0, T])} \leq K \lambda^{\gamma_{c}+\frac{\sigma(4-d) \gamma_{c}}{2(d-5+4 \sigma)}}
$$

for some constant $K>0$. This contradicts with (1.12) by taking $C$ larger than $2 \mathrm{~K}$. We thus obtain (1.8) and also

$$
E\left(I u_{\lambda}(t)\right) \leq 1, \quad \forall t \in[0, \infty) .
$$

This also gives the uniform bound (1.9). In order to prove the scattering property, we will upgrade the uniform Morawetz bound (1.8) to the uniform Strichartz bound, namely

$$
\|u\|_{S^{\gamma}(\mathbb{R})}:=\sup _{(p, q) \in B}\left\|\langle\nabla\rangle^{\gamma} u\right\|_{L_{t}^{p}\left(\mathbb{R}, L_{x}^{q}\right)} \leq C\left(\left\|u_{0}\right\|_{H_{x}^{\gamma}}\right) .
$$

Here $(p, q) \in B$ means that $(p, q)$ is biharmonic admissible (see again Section 2 for the definition). With this uniform Strichartz bound, the scattering property follows by a standard argument. We refer the reader to Section 4 for more details.

This paper is organized as follows. We firstly introduce some notations and recall some results related to our problem in Section 2. In Section 3, we show the almost conservation law for the modified energy. Finally, we give the proof of our main result in Section 4.

\section{Preliminaries}

In the sequel, the notation $A \lesssim B$ denotes an estimate of the form $A \leq C B$ for some constant $C>0$. The notation $A \sim B$ means that $A \lesssim B$ and $B \lesssim A$. We write $A \ll B$ if $A \leq c B$ for some small constant $c>0$. We also use $\langle a\rangle:=1+|a|$. 
V. D. DINH

2.1. Nonlinearity. Let $F(z):=|z|^{\nu-1} z$ be the function which defines the nonlinearity in (NL4S). The derivative of $F(z)$ is defined by

$$
F^{\prime}(z):=\left(\partial_{z} F(z), \partial_{\bar{z}} F(z)\right),
$$

where

$$
\partial_{z} F(z)=\frac{\nu+1}{2}|z|^{\nu-1}, \quad \partial_{\bar{z}} F(z)=\frac{\nu-1}{2}|z|^{\nu-1} \frac{z}{\bar{z}} .
$$

We also define its norm as

$$
\left|F^{\prime}(z)\right|:=\left|\partial_{z} F(z)\right|+\left|\partial_{\bar{z}} F(z)\right| .
$$

It is clear that $\left|F^{\prime}(z)\right|=O\left(|z|^{\nu-1}\right)$. For a complex-valued function $u$, we have the following chain rule

$$
\partial_{k} F(u)=F^{\prime}(u) \partial_{k} u
$$

for $k \in\{1, \cdots, d\}$. In particular,

$$
\nabla F(u)=F^{\prime}(u) \nabla u
$$

In order to estimate the nonlinearity, we need to recall the following fractional chain rules.

Lemma 2.1 ([CW91], [KPV93]). Suppose that $G \in C^{1}(\mathbb{C}, \mathbb{C})$, and $\alpha \in(0,1)$. Then for $1<q \leq$ $q_{2}<\infty$ and $1<q_{1} \leq \infty$ satisfying $\frac{1}{q}=\frac{1}{q_{1}}+\frac{1}{q_{2}}$,

$$
\left\||\nabla|^{\alpha} G(u)\right\|_{L_{x}^{q}} \lesssim\left\|G^{\prime}(u)\right\|_{L_{x}^{q_{1}}}\left\||\nabla|^{\alpha} u\right\|_{L_{x}^{q_{2}}} .
$$

Lemma 2.2 ([Vis06]). Suppose that $G \in C^{0, \beta}(\mathbb{C}, \mathbb{C}), \beta \in(0,1)$. Then for every $0<\alpha<\beta, 1<$ $q<\infty$, and $\frac{\alpha}{\beta}<\rho<1$,

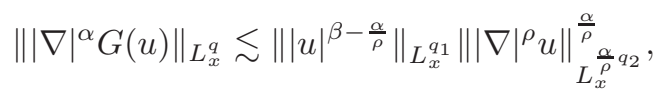

provided $\frac{1}{q}=\frac{1}{q_{1}}+\frac{1}{q_{2}}$ and $\left(1-\frac{\alpha}{\beta \rho}\right) q_{1}>1$.

The reader can find the proof of Lemma 2.1 in the case $1<q_{1}<\infty$ in [CW91, Proposition 3.1] and [KPV93, Theorem A.6] when $q_{1}=\infty$. For the proof of Lemma 2.2, we refer to [Vis06, Proposition A.1].

2.2. Strichartz estimates. Let $I \subset \mathbb{R}$ and $p, q \in[1, \infty]$. The Strichartz norm is defined as

$$
\|u\|_{L_{t}^{p}\left(I, L_{x}^{q}\right)}:=\left(\int_{I}\left(\int_{\mathbb{R}^{d}}|u(t, x)|^{q} d x\right)^{\frac{1}{q}}\right)^{\frac{1}{p}}
$$

with a usual modification when either $p$ or $q$ are infinity. When there is no risk of confusion, we write $L_{t}^{p} L_{x}^{q}$ instead of $L_{t}^{p}\left(I, L_{x}^{q}\right)$. When $p=q$, we also use $L_{t, x}^{p}$.

Definition 2.3. A pair $(p, q)$ is said to be Schrödinger admissible, for short $(p, q) \in S$, if

$$
(p, q) \in[2, \infty]^{2}, \quad(p, q, d) \neq(2, \infty, 2), \quad \frac{2}{p}+\frac{d}{q} \leq \frac{d}{2} .
$$

We denote for $(p, q) \in[1, \infty]^{2}$,

$$
\gamma_{p, q}=\frac{d}{2}-\frac{d}{q}-\frac{4}{p}
$$

Definition 2.4. A pair $(p, q)$ is called biharmonic admissible, for short $(p, q) \in B$, if

$$
(p, q) \in S, \quad \gamma_{p, q}=0 .
$$


Proposition 2.5 (Strichartz estimates for the fourth-order Schrödinger equation [Din1]). Let $\gamma \in \mathbb{R}$ and $u$ be a (weak) solution to the linear fourth-order Schrödinger equation, namely

$$
u(t)=e^{i t \Delta^{2}} u_{0}+\int_{0}^{t} e^{i(t-s) \Delta^{2}} F(s) d s,
$$

for some data $u_{0}, F$. Then for all $(p, q)$ and $(a, b)$ Schrödinger admissible with $q<\infty$ and $b<\infty$,

$$
\left\||\nabla|^{\gamma} u\right\|_{L_{t}^{p}\left(\mathbb{R}, L_{x}^{q}\right)} \lesssim\left\||\nabla|^{\gamma+\gamma_{p, q}} u_{0}\right\|_{L_{x}^{2}}+\left\||\nabla|^{\gamma+\gamma_{p, q}-\gamma_{a^{\prime}, b^{\prime}}-4} F\right\|_{L_{t}^{a^{\prime}}\left(\mathbb{R}, L_{x}^{b^{\prime}}\right)} .
$$

Here $\left(a, a^{\prime}\right)$ and $\left(b, b^{\prime}\right)$ are conjugate pairs, and $\gamma_{p, q}, \gamma_{a^{\prime}, b^{\prime}}$ are defined as in (2.2).

The estimate (2.3) is exactly the one given in [MZ07], [Pau1] or [Pau2] where the author considered $(p, q)$ and $(a, b)$ are either sharp Schrödinger admissible, i.e.

$$
p, q \in[2, \infty]^{2}, \quad(p, q, d) \neq(2, \infty, 2), \quad \frac{2}{p}+\frac{d}{q}=\frac{d}{2},
$$

or biharmonic admissible. We refer the reader to [Din1, Proposition 2.1] for the proof of Proposition 2.5. The proof is based on the scaling technique instead of using a dedicate dispersive estimate of [BKS00] for the fundamental solution of the homogeneous fourth-order Schrödinger equation.

The following result is a direct consequence of (2.3).

Corollary 2.6. Let $\gamma \in \mathbb{R}$ and $u$ be a (weak) solution to the linear fourth-order Schrödinger equation for some data $u_{0}, F$. Then for all $(p, q)$ and $(a, b)$ biharmonic admissible satisfying $q<\infty$ and $b<\infty$,

$$
\|u\|_{L_{t}^{p}\left(\mathbb{R}, L_{x}^{q}\right)} \lesssim\left\|u_{0}\right\|_{L_{x}^{2}}+\|F\|_{L_{t}^{a^{\prime}}\left(\mathbb{R}, L_{x}^{b^{\prime}}\right)}
$$

and

$$
\left\||\nabla|^{\gamma} u\right\|_{L_{t}^{p}\left(\mathbb{R}, L_{x}^{q}\right)} \lesssim\left\||\nabla|^{\gamma} u_{0}\right\|_{L_{x}^{2}}+\left\||\nabla|^{\gamma-1} F\right\|_{L_{t}^{2}\left(\mathbb{R}, L_{x}^{\frac{2 d}{d+2}}\right)} .
$$

2.3. Littlewood-Paley decomposition. Let $\varphi$ be a radial smooth bump function supported in the ball $|\xi| \leq 2$ and equal to 1 on the ball $|\xi| \leq 1$. For $M=2^{k}, k \in \mathbb{Z}$, we define the LittlewoodPaley operators

$$
\begin{aligned}
\widehat{P_{\leq M} f}(\xi) & :=\varphi\left(M^{-1} \xi\right) \hat{f}(\xi), \\
\widehat{P_{>M} f}(\xi) & :=\left(1-\varphi\left(M^{-1} \xi\right)\right) \hat{f}(\xi), \\
\widehat{P_{M} f}(\xi) & :=\left(\varphi\left(M^{-1} \xi\right)-\varphi\left(2 M^{-1} \xi\right)\right) \hat{f}(\xi),
\end{aligned}
$$

where $\hat{\cdot}$ is the spatial Fourier transform. Similarly, we can define for $M, M_{1} \leq M_{2} \in 2^{\mathbb{Z}}$,

$$
P_{<M}:=P_{\leq M}-P_{M}, \quad P_{\geq M}:=P_{>M}+P_{M}, \quad P_{M_{1}<\leq M_{2}}:=P_{\leq M_{2}}-P_{\leq M_{1}}=\sum_{M_{1}<M \leq M_{2}} P_{M} .
$$

We recall the following standard Bernstein inequalities (see e.g. [BCD11, Chapter 2] or [Tao06, Appendix]).

Lemma 2.7 (Bernstein inequalities). Let $\gamma \geq 0$ and $1 \leq p \leq q \leq \infty$. We have

$$
\begin{aligned}
\left\|P_{\geq M} f\right\|_{L_{x}^{p}} & \lesssim M^{-\gamma}\left\||\nabla|^{\gamma} P_{\geq M} f\right\|_{L_{x}^{p}}, \\
\left\|P_{\leq M}|\nabla|^{\gamma} f\right\|_{L_{x}^{p}} & \lesssim M^{\gamma}\left\|P_{\leq M} f\right\|_{L_{x}^{p}}, \\
\left\|P_{M}|\nabla|^{ \pm \gamma} f\right\|_{L_{x}^{p}} & \sim M^{ \pm \gamma}\left\|P_{M} f\right\|_{L_{x}^{p}}, \\
\left\|P_{\leq M} f\right\|_{L_{x}^{q}} & \lesssim M^{\frac{d}{p}-\frac{d}{q}}\left\|P_{\leq M} f\right\|_{L_{x}^{p}} \\
\left\|P_{M} f\right\|_{L_{x}^{q}} & \lesssim M^{\frac{d}{p}-\frac{d}{q}}\left\|P_{M} f\right\|_{L_{x}^{p}}
\end{aligned}
$$


V. D. DINH

2.4. $I$-operator. Let $0 \leq \gamma<2$ and $N \gg 1$. We define the Fourier multiplier $I_{N}$ by

$$
\widehat{I_{N} f}(\xi):=m_{N}(\xi) \hat{f}(\xi)
$$

where $m_{N}$ is a smooth, radially symmetric, non-increasing function such that

$$
m_{N}(\xi):=\left\{\begin{array}{cl}
1 & \text { if }|\xi| \leq N \\
\left(N^{-1}|\xi|\right)^{\gamma-2} & \text { if }|\xi| \geq 2 N
\end{array}\right.
$$

We shall drop the $N$ from the notation and write $I$ and $m$ instead of $I_{N}$ and $m_{N}$. We collect some basic properties of the $I$-operator in the following lemma.

Lemma 2.8 ([Din3]). Let $0 \leq \sigma \leq \gamma<2$ and $1<q<\infty$. Then

$$
\begin{aligned}
\|I f\|_{L_{x}^{q}} & \lesssim\|f\|_{L_{x}^{q}}, \\
\left\||\nabla|^{\sigma} P_{>N} f\right\|_{L_{x}^{q}} & \lesssim N^{\sigma-2}\|\Delta I f\|_{L_{x}^{q}}, \\
\left\|\langle\nabla\rangle^{\sigma} f\right\|_{L_{x}^{q}} & \lesssim\|\langle\Delta\rangle I f\|_{L_{x}^{q},} \\
\|f\|_{H_{x}^{\gamma}} \lesssim\|I f\|_{H_{x}^{2}} & \lesssim N^{2-\gamma}\|f\|_{H_{x}^{\gamma}}, \\
\|I f\|_{H_{x}^{2}} & \lesssim N^{2-\gamma}\|f\|_{\dot{H}_{x}^{\gamma}} .
\end{aligned}
$$

We refer to [Din3, Lemma 2.7] for the proof of these estimates. We also recall the following product rule which is a modified version of the one given in [VZ09, Lemma 2.5] in the context of nonlinear Schrödinger equation.

Lemma 2.9 ([Din3]). Let $\gamma>1,0<\delta<\gamma-1$ and $1<q, q_{1}, q_{2}<\infty$ be such that $\frac{1}{q}=\frac{1}{q_{1}}+\frac{1}{q_{2}}$. Then

$$
\|I(f g)-(I f) g\|_{L_{x}^{q}} \lesssim N^{-(2-\gamma+\delta)}\|I f\|_{L_{x}^{q_{1}}}\left\|\langle\nabla\rangle^{2-\gamma+\delta} g\right\|_{L_{x}^{q_{2}}} .
$$

We again refer the reader to [Din3, Lemma 2.8] for the proof of this lemma. A direct consequence of Lemma 2.9 and (2.1) is the following corollary.

Corollary 2.10. Let $\gamma>1,0<\delta<\gamma-1$ and $1<q, q_{1}, q_{2}<\infty$ be such that $\frac{1}{q}=\frac{1}{q_{1}}+\frac{1}{q_{2}}$. Then

$$
\left\|\nabla I F(u)-(I \nabla u) F^{\prime}(u)\right\|_{L_{x}^{q}} \lesssim N^{-(2-\gamma+\delta)}\|\nabla I u\|_{L_{x}^{q_{1}}}\left\|\langle\nabla\rangle^{2-\gamma+\delta} F^{\prime}(u)\right\|_{L_{x}^{q_{2}}} .
$$

2.5. Interaction Morawetz inequality. We now recall the interaction Morawetz inequality for the nonlinear fourth-order Schrödinger equation.

Proposition 2.11 (Interaction Morawetz inequality [Pau2], [MWZ15]). Let $d \geq 5, J$ be a compact time interval and $u$ a solution to (NL4S) on the spacetime slab $J \times \mathbb{R}^{d}$. Then we have the following a priori estimate:

$$
\left\||\nabla|^{-\frac{d-5}{4}} u\right\|_{L_{t}^{4}\left(J, L_{x}^{4}\right)} \lesssim\left\|u_{0}\right\|_{L_{x}^{2}}^{\frac{1}{2}}\|u\|_{L_{t}^{\infty}\left(J, \dot{H}_{x}^{\frac{1}{2}}\right)}^{\frac{1}{2}} .
$$

This estimate was first established by Pausader in [Pau2] for $d \geq 7$. Later, Miao-Wu-Zhang in [MWZ15] extended this interaction Morawetz estimate to $d \geq 5$. By interpolating (2.13) and the trivial estimate for $0<\sigma \leq \gamma$,

we obtain

$$
\|u\|_{L_{t}^{\infty}\left(J, \dot{H}_{x}^{\sigma}\right)} \leq\|u\|_{L_{t}^{\infty}\left(J, \dot{H}_{x}^{\sigma}\right)}
$$

$$
\|u\|_{M^{\sigma}(J)} \lesssim\left(\left\|u_{0}\right\|_{L_{x}^{2}}\|u\|_{L_{t}^{\infty}\left(J, \dot{H}_{x}^{\frac{1}{2}}\right)}\right)^{\frac{2 \sigma}{d-5+4 \sigma}}\|u\|_{L_{t}^{\infty}\left(J, \dot{H}_{x}^{\sigma}\right)}^{\frac{d-5}{d-5+4 \sigma}},
$$

where

$$
\|u\|_{M^{\sigma}(J)}:=\|u\|_{L_{t}^{\frac{d-5+4 \sigma}{\sigma}}} L_{x}^{\frac{2(d-5+4 \sigma)}{d-5+2 \sigma}} .
$$




\section{Almost conservation LaW}

For any spacetime slab $J \times \mathbb{R}^{d}$, we define

$$
Z_{I}(J):=\sup _{(p, q) \in B}\|\langle\Delta\rangle I u\|_{L_{t}^{p}\left(J, L_{x}^{q}\right)}
$$

Note that in our considerations, the biharmonic admissible condition $(p, q) \in B$ ensures $q<\infty$. Let us start with the following commutator estimates.

Lemma 3.1. Let $5 \leq d \leq 11, \frac{2+\gamma_{c}}{2}<\gamma<2,0<\delta<\min \left\{2 \gamma-\gamma_{c}-2, \gamma-1\right\}, 0<\sigma \leq \gamma$ and

$$
\max \left\{\frac{8(d-5+4 \sigma)}{d(d-5+2 \sigma)+8 \sigma}, 1\right\}<\nu-1<\min \left\{\frac{d-5+4 \sigma}{2 \sigma}, \frac{8}{d-2 \gamma}\right\} \text {. }
$$

Assume that

$$
\|u\|_{M^{\sigma}(J)} \leq \mu
$$

for some small constant $\mu>0$. Then

$$
\begin{aligned}
\left\|\nabla I F(u)-(I \nabla u) F^{\prime}(u)\right\|_{L_{t}^{2}\left(J, L_{x}^{\frac{2 d}{d+2}}\right)} & \lesssim N^{-(2-\gamma+\delta)} Z_{I}(J)\left(\mu^{\theta} Z_{I}^{1-\theta}(J)+Z_{I}(J)\right)^{\nu-1} \\
\|\nabla I F(u)\|_{L_{t}^{2}\left(J, L_{x}^{\frac{2 d}{d+2}}\right)} & \lesssim N^{-(2-\gamma+\delta)} Z_{I}^{\nu}(J)+\mu^{(\nu-1) \theta} Z_{I}^{1+(\nu-1)(1-\theta)}(J),
\end{aligned}
$$

where

$$
\theta:=\frac{(d-5+4 \sigma)(8-(d-4)(\nu-1))}{2(\nu-1)(2(d-5)+(12-d) \sigma)} \in(0,1)
$$

Proof. For simplifying the presentation, we shall drop the dependence on the time interval $J$. Denote

$$
\varepsilon:=\frac{4(\nu-1) \sigma}{d-5+4 \sigma-2(\nu-1) \sigma} .
$$

It is easy to see from our assumptions that $\varepsilon>0$. We next apply (2.12) with $q=\frac{2 d}{d+2}, q_{1}=$ $\frac{2 d(2+\varepsilon)}{(d-2)(2+\varepsilon)-8}$ and $q_{2}=\frac{d(2+\varepsilon)}{2 \varepsilon+8}$ to get

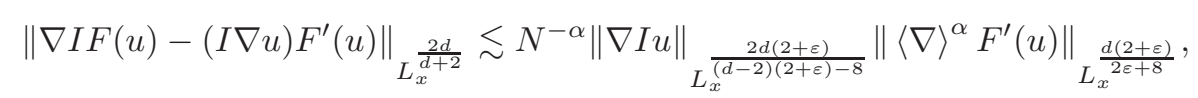

where $\alpha=2-\gamma+\delta$. Note that $q_{1}$ is well-defined since $(d-2)(2+\varepsilon)-8>0$. We then apply Hölder's inequality in time to have

$$
\left\|\nabla I F(u)-(I \nabla u) F^{\prime}(u)\right\|_{L_{t}^{2} L_{x}^{\frac{2 d}{d+2}}} \lesssim N^{-\alpha}\|\nabla I u\|_{L_{t}^{2+\varepsilon} L_{x}^{(d-2)(2+\varepsilon)-8}}\left\|\langle\nabla\rangle^{\alpha} F^{\prime}(u)\right\|_{L_{t} \frac{2(2+\varepsilon)}{\varepsilon}} \frac{d(2+\varepsilon)}{L_{x}^{2 \varepsilon+8}} .
$$

For the first factor in the right hand side of (3.4), we use the Sobolev embedding to obtain

$$
\|\nabla I u\|_{L_{t}^{2+\varepsilon} L_{x}^{\frac{2 d(2+\varepsilon)}{(d-2)(2+\varepsilon)-8}}} \lesssim\|\Delta I u\|_{L_{t}^{2+\varepsilon} L_{x}^{\frac{2 d(2+\varepsilon)}{d(2+\varepsilon)-8}}} \lesssim Z_{I},
$$

where $\left(2+\varepsilon, \frac{2 d(2+\varepsilon)}{d(2+\varepsilon)-8}\right)$ is a biharmonic admissible pair. To treat the second factor in the right hand side of (3.4), we note that $\alpha<\gamma-\gamma_{\mathrm{c}}$ by our assumption on $\delta$. Thus

$$
\begin{aligned}
& \left\|\langle\nabla\rangle^{\alpha} F^{\prime}(u)\right\|_{L_{t}^{\frac{2(2+\varepsilon)}{\varepsilon}}} \frac{d(2+\varepsilon)}{L_{x}^{2 \varepsilon+8}} \lesssim\left\|\langle\nabla\rangle^{\gamma-\gamma_{c}} F^{\prime}(u)\right\|_{L_{t}^{\frac{2(2+\varepsilon)}{\varepsilon}}} \frac{d(2+\varepsilon)}{L_{x}^{2 \varepsilon+8}} \\
& \lesssim\left\|F^{\prime}(u)\right\|_{L_{t}^{\frac{2(2+\varepsilon)}{\varepsilon}}} L_{x}^{\frac{d(2+\varepsilon)}{2 \varepsilon+8}}+\left\||\nabla|^{\gamma-\gamma_{\mathrm{c}}} F^{\prime}(u)\right\|_{L_{t}^{\frac{2(2+\varepsilon)}{\varepsilon}}{ }_{L_{x}}^{\frac{d(2+\varepsilon)}{2 \varepsilon+8}}} .
\end{aligned}
$$


Since $F^{\prime}(u)=O\left(|u|^{\nu-1}\right)$, we bound the first term in (3.6) as

$$
\left\|F^{\prime}(u)\right\|_{L_{t}^{\frac{2(2+\varepsilon)}{\varepsilon}}} L_{x}^{\frac{d(2+\varepsilon)}{2 \varepsilon+8}} \lesssim\|u\|_{L_{t}^{\frac{2(\nu-1)(2+\varepsilon)}{\varepsilon}}}^{\frac{2(\nu-1)(2+\varepsilon)}{2 \varepsilon+8}} .
$$

By the choice of $\varepsilon$, we have

$$
\frac{2(\nu-1)(2+\varepsilon)}{\varepsilon}=\frac{d-5+4 \sigma}{\sigma}, \quad \frac{d(\nu-1)(2+\varepsilon)}{2 \varepsilon+8}=\frac{d(\nu-1)(d-5+4 \sigma)}{4(d-5+4 \sigma-(\nu-1) \sigma)} .
$$

We next split $u:=P_{\leq N} u+P_{>N} u$. For the low frequency part, we estimate

$$
\begin{aligned}
& \left\|P_{\leq N} u\right\|_{L_{t}^{\frac{d-5+4 \sigma}{\sigma}}} \frac{\frac{d(\nu-1)(d-5+4 \sigma)}{4(d-5+4 \sigma-(\nu-1) \sigma)}}{L_{x}} \lesssim\left\|P_{\leq N} u\right\|_{M^{\sigma}}^{\theta}\left\|P_{\leq N} u\right\|_{L_{t}^{\frac{d-5+4 \sigma}{\sigma}}}^{1-\theta}{ }_{L_{x}^{(d-4)(d-5+4 \sigma)-8 \sigma}} \\
& \lesssim\left\|P_{\leq N} u\right\|_{M^{\sigma}}^{\theta}\left\|\Delta P_{\leq N} u\right\|_{L_{t}^{\frac{d-\theta+4+}{\sigma}}}^{\frac{1-\theta}{\frac{d(d(d-5+4 \sigma)}{d(d-5+4 \sigma)-8 \sigma}}} \\
& \lesssim \mu^{\theta} Z_{I}^{1-\theta}
\end{aligned}
$$

where $\theta$ is given in (3.3). Here the first line follows from Hölder's inequality, and the second line makes use of the Sobolev embedding. The last inequality uses the fact that $\left(\frac{d-5+4 \sigma}{\sigma}, \frac{2 d(d-5+4 \sigma)}{d(d-5+4 \sigma)-8 \sigma}\right)$ is biharmonic admissible. Note that our assumptions ensure $\theta \in(0,1)$. For the high frequency part, the Sobolev embedding gives

$$
\left\|P_{>N} u\right\|_{L_{t}^{\frac{d-5+4 \sigma}{\sigma}}} \frac{d(\nu-1)(d-5+4 \sigma)}{L_{x}^{4(d-5+4 \sigma-(\nu-1) \sigma)}} \lesssim\left\||\nabla|^{\gamma_{c}} P_{>N} u\right\|_{L_{t}^{\frac{d-5+4 \sigma}{\sigma}}} L_{x}^{\frac{2 d(d-5+4 \sigma)}{d(d-5+4 \sigma)-8 \sigma}} \lesssim N^{\gamma_{c}-2} Z_{I}
$$

Here $\left(\frac{d-5+4 \sigma}{\sigma}, \frac{2 d(d-5+4 \sigma)}{d(d-5+4 \sigma)-8 \sigma}\right)$ is biharmonic admissible. Thus, we obtain

$$
\|u\|_{L_{t}^{\frac{2(\nu-1)(2+\varepsilon)}{\varepsilon}}} \frac{d(\nu-1)(2+\varepsilon)}{2 \varepsilon+8} \lesssim \mu^{\theta} Z_{I}^{1-\theta}+N^{\gamma_{c}-2} Z_{I} .
$$

In particular,

$$
\left\|F^{\prime}(u)\right\|_{L_{t}^{\frac{2(2+\varepsilon)}{\varepsilon}}} \frac{d(2+\varepsilon)}{L_{x}^{\varepsilon+8}} \lesssim\left(\mu^{\theta} Z_{I}^{1-\theta}+Z_{I}\right)^{\nu-1} .
$$

We next treat the second term in (3.6). Since $\nu-1>1$, we are able to apply Lemma 2.1 to get

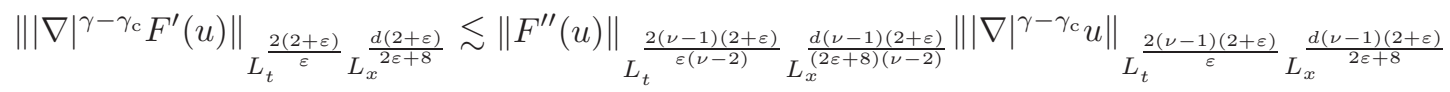

$$
\begin{aligned}
& \lesssim\|u\|_{L_{t} \frac{2(\nu-1)(2+\varepsilon)}{\varepsilon}}^{\frac{2-2}{\varepsilon}} L_{x}^{\frac{d(\nu-1)(2+\varepsilon)}{2 \varepsilon+8}}\left\||\nabla|^{\gamma-\gamma_{c}} u\right\|_{L_{t}^{\frac{2(\nu-1)(2+\varepsilon)}{\varepsilon}} L_{x}}^{\frac{d(\nu-1)(2+\varepsilon)}{2 \varepsilon+8}},
\end{aligned}
$$

where $F^{\prime \prime}(u)=O\left(|u|^{\nu-2}\right)$. The first factor in the right hand side of (3.11) is treated in (3.9). For the second factor, we split $u:=P_{\leq 1} u+P_{1<\cdot \leq N} u+P_{>N} u$. We use Bernstein inequality and estimate as in (3.7),

$$
\left\||\nabla|^{\gamma-\gamma_{c}} P_{\leq 1} u\right\|_{L_{t} \frac{2(\nu-1)(2+\varepsilon)}{\varepsilon}} L_{x}^{\frac{d(\nu-1)(2+\varepsilon)}{2 \varepsilon+8}} \lesssim\left\|P_{\leq 1} u\right\|_{L_{t}^{\frac{2(\nu-1)(2+\varepsilon)}{\varepsilon}}} L_{x}^{\frac{d(\nu-1)(2+\varepsilon)}{2 \varepsilon+8}} \lesssim \mu^{\theta} Z_{I}^{1-\theta} .
$$

The intermediate term is bounded by

$$
\begin{aligned}
& \left\||\nabla|^{\gamma-\gamma_{c}} P_{1<\cdot \leq N} u\right\|_{L_{t}^{\frac{2(\nu-1)(2+\varepsilon)}{\varepsilon}} L_{x} \frac{d(\nu-1)(2+\varepsilon)}{2 \varepsilon+8}} \lesssim\left\||\nabla|^{\gamma} P_{1<\cdot \leq N} u\right\|_{L_{t}^{\frac{2(\nu-1)(2+\varepsilon)}{\varepsilon}}} L_{x}^{\frac{2 d(\nu-1)(2+\varepsilon)}{d(\nu-1)(2+\varepsilon)-4 \varepsilon}} \\
& \lesssim\left\|\Delta I P_{1<\cdot \leq N} u\right\|_{L_{t}^{\frac{2(\nu-1)(2+\varepsilon)}{\varepsilon}}} L_{x}^{\frac{2 d(\nu-1)(2+\varepsilon)}{d(\nu-1)(2+\varepsilon)-4 \varepsilon}} \\
& \lesssim Z_{I}
\end{aligned}
$$


Here we use

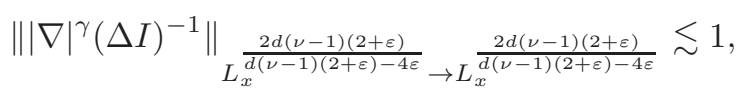

and the fact $\left(\frac{2(\nu-1)(2+\varepsilon)}{\varepsilon}, \frac{2 d(\nu-1)(2+\varepsilon)}{d(\nu-1)(2+\varepsilon)-4 \varepsilon}\right)$ is biharmonic admissible. Finally, we use (2.7) to estimate

$$
\begin{aligned}
& \left\||\nabla|^{\gamma-\gamma_{c}} P_{>N} u\right\|_{L_{t} \frac{2(\nu-1)(2+\varepsilon)}{\varepsilon}} \frac{d(\nu-1)(2+\varepsilon)}{2 \varepsilon+8} \lesssim\left\||\nabla|^{\gamma} P_{>N} u\right\|_{L_{t} \frac{2(\nu-1)(2+\varepsilon)}{\varepsilon}} \frac{2 d(\nu-1)(2+\varepsilon)}{L_{x}^{((\nu-1)(2+\varepsilon)-4 \varepsilon}} \\
& \lesssim N^{\gamma-2}\left\|\Delta P_{>N} u\right\|_{L_{t}^{\frac{2(\nu-1)(2+\varepsilon)}{\varepsilon}}} \frac{2 d(\nu-1)(2+\varepsilon)}{L_{x}^{d(\nu-1)(2+\varepsilon)-4 \varepsilon}} \\
& \lesssim N^{\gamma-2} Z_{I}
\end{aligned}
$$

Combining three terms yields

$$
\left\||\nabla|^{\gamma-\gamma_{c}} u\right\|_{L_{t}^{\frac{2(2+\varepsilon)}{\varepsilon}}} \frac{d(\nu-1)(2+\varepsilon)}{2 \varepsilon+8} \lesssim \mu^{\theta} Z_{I}^{1-\theta}+Z_{I}
$$

Collecting (3.4), (3.5), (3.10), (3.11) and (3.12), we show the first estimate (3.1).

We now prove (3.2). By triangle inequality,

$$
\|I \nabla F(u)\|_{L_{t}^{2} L_{x}^{\frac{2 d}{d+2}}} \leq\left\|(I \nabla u) F^{\prime}(u)\right\|_{L_{t}^{2} L_{x}^{\frac{2 d}{d+2}}}+\left\|I \nabla F(u)-(I \nabla u) F^{\prime}(u)\right\|_{L_{t}^{2} L_{x}^{\frac{2 d}{d+2}}} .
$$

We have from Hölder's inequality, (3.5) and (3.9) that

$$
\begin{aligned}
\left\|(I \nabla u) F^{\prime}(u)\right\|_{L_{t}^{2} L_{x}^{\frac{2 d}{d+2}}} & \lesssim\|I \nabla u\| L_{L_{t}^{2+\varepsilon}} \frac{2 d(2+\varepsilon)}{L_{x}^{(d-2)(2+\varepsilon)-8}}\left\|F^{\prime}(u)\right\|{ }_{L_{t}^{\frac{2(2+\varepsilon)}{\varepsilon}}} \frac{d(2+\varepsilon)}{L_{x}^{2 \varepsilon+8}} \\
& \lesssim\|\Delta I u\|{ }_{L_{t}^{2+\varepsilon} \frac{2 d(2+\varepsilon)}{d(2+\varepsilon)-8}\|u\|^{\nu-1} \frac{2(\nu-1)(2+\varepsilon)}{L_{t}}} \frac{d(\nu-1)(2+\varepsilon)}{L_{x}} \\
& \lesssim Z_{I}\left(\mu^{\theta} Z_{I}^{1-\theta}+N^{\gamma_{c}-2} Z_{I}\right)^{\nu-1} \\
& \lesssim \mu^{\theta(\nu-1)} Z_{I}^{1+(1-\theta)(\nu-1)}+N^{\left(\gamma_{c}-2\right)(\nu-1)} Z_{I}^{\nu}
\end{aligned}
$$

The estimate (3.2) follows easily from (3.1) and (3.13). Note that by our assumptions, $\alpha=$ $2-\gamma+\delta<\gamma-\gamma_{\mathrm{c}}<2-\gamma_{\mathrm{c}}$. The proof is complete.

Remark 3.2. The estimates (3.1) and (3.2) still hold for $\nu-1=\frac{d-5+4 \sigma}{2 \sigma}$. Indeed, the proof of Lemma 3.1 is valid for $\varepsilon=\infty$.

We are now able to prove the almost conservation law for the modified energy functional $E(I u)$, where

$$
E(I u(t))=\frac{1}{2}\|I u(t)\|_{\dot{H}_{x}^{2}}^{2}+\frac{1}{\nu+1}\|I u(t)\|_{L_{x}^{\nu+1}}^{\nu+1} .
$$

Proposition 3.3. Let $5 \leq d \leq 11$,

$$
\begin{gathered}
\max \left\{\frac{3}{2}+\frac{\gamma_{\mathrm{c}}}{4}, 4-\nu, \frac{2}{\nu-1}+\frac{(\nu-2) \gamma_{\mathrm{c}}}{\nu-1}\right\}<\gamma<2, \\
0<\delta<\min \left\{2 \gamma-3-\frac{\gamma_{\mathrm{c}}}{2}, \gamma+\nu-4,(\nu-1) \gamma-2-(\nu-2) \gamma_{\mathrm{c}}\right\}, 0<\sigma \leq \gamma \text { and } \\
\max \left\{\frac{8(d-5+4 \sigma)}{d(d-5+2 \sigma)+8 \sigma}, 1\right\}<\nu-1<\min \left\{\frac{d-5+4 \sigma}{2 \sigma}, \frac{8}{d-2 \gamma}\right\} .
\end{gathered}
$$

Assume that $u \in L^{\infty}\left([0, T], \mathscr{S}\left(\mathbb{R}^{d}\right)\right)$ is a solution to (NL4S) on a time interval $[0, T]$, and satisfies $\left\|I u_{0}\right\|_{H_{x}^{2}} \leq 1$. Assume in addition that $u$ satisfies the a priori bound

$$
\|u\|_{M^{\sigma}([0, T])} \leq \mu
$$


for some small constant $\mu>0$. Then, for $N$ sufficiently large,

$$
\sup _{t \in[0, T]}\left|E(I u(t))-E\left(I u_{0}\right)\right| \lesssim N^{-(2-\gamma+\delta)} .
$$

Here the implicit constant depends only on the size of $E\left(I u_{0}\right)$.

Remark 3.4. As in Remark 3.2, the estimate (3.14) is still valid for $\nu-1=\frac{d-5+4 \sigma}{2 \sigma}$.

Proof of Proposition 3.3. We firstly note that our assumptions on $\gamma$ and $\delta$ satisfy the assumptions given in Lemma 3.1. It allows us to use the estimates given in Lemma 3.1.

We begin by controlling the size of $Z_{I}$. By applying $I, \Delta I$ to (NL4S), and using Strichartz estimates $(2.4),(2.5)$, we get

$$
Z_{I} \lesssim\left\|I u_{0}\right\|_{H_{x}^{2}}+\|I F(u)\|_{L_{t}^{2} L_{x}^{\frac{2 d}{d+4}}}+\|\nabla I F(u)\|_{L_{t}^{2} L_{x}^{\frac{2 d}{d+2}}} .
$$

Using (3.2), we have

$$
\|\nabla I F(u)\|_{L_{t}^{2} L_{x}^{\frac{2 d}{d+2}}} \lesssim N^{-(2-\gamma+\delta)} Z_{I}^{\nu}+\mu^{(\nu-1) \theta} Z_{I}^{1+(\nu-1)(1-\theta)} .
$$

Next, we drop the I-operator and use Hölder's inequality together with (3.9) to estimate

$$
\begin{aligned}
\|I F(u)\|_{L_{t}^{2} L_{x}^{\frac{2 d}{d+4}}} & \lesssim\left\|\left.u\right|^{\nu-1}\right\|_{L_{t}^{\frac{2(2+\varepsilon)}{\varepsilon}}} \frac{\frac{d(2+\varepsilon)}{L_{x}^{2 \varepsilon+8}}\|u\|}{L_{t}^{2+\varepsilon}} L_{x}^{\frac{2 d(2+\varepsilon)}{d(2+\varepsilon)-8}} \\
& \lesssim\|u\|^{\nu-1} L_{t}^{\frac{2(\nu-1)(2+\varepsilon)}{\varepsilon}} L_{x}^{\frac{d(\nu-1)(2+\varepsilon)}{2 \varepsilon+8}}\|u\| \\
& \lesssim Z_{I}\left(\mu^{\theta} Z_{I}^{1-\theta}+N_{x}^{\frac{2 d(2+\varepsilon)}{d(2+\varepsilon)-8}}\right. \\
& \left.\lesssim \mu^{(\nu-1) \theta} Z_{I}^{\gamma_{c}-2} Z_{I}\right)^{\nu-1}
\end{aligned}
$$

Here $\left(2+\varepsilon, \frac{2 d(2+\varepsilon)}{d(2+\varepsilon)-8}\right)$ is biharmonic admissible. We thus get

$$
Z_{I} \lesssim\left\|I u_{0}\right\|_{H_{x}^{2}}+N^{-(2-\gamma+\delta)} Z_{I}^{\nu}+\mu^{(\nu-1) \theta} Z_{I}^{1+(\nu-1)(1-\theta)} .
$$

By taking $\mu$ sufficiently small and $N$ sufficiently large and using the assumption $\left\|I u_{0}\right\|_{H_{x}^{2}} \leq 1$, the continuity argument gives

$$
Z_{I} \lesssim\left\|I u_{0}\right\|_{H_{x}^{2}} \leq 1
$$

Now, let $F(u)=|u|^{\nu-1} u$. A direct computation shows

$$
\partial_{t} E(I u(t))=\operatorname{Re} \int \overline{I \partial_{t} u}\left(\Delta^{2} I u+F(I u)\right) d x .
$$

By the Fundamental Theorem of Calculus,

$$
E(I u(t))-E\left(I u_{0}\right)=\int_{0}^{t} \partial_{s} E(I u(s)) d s=\operatorname{Re} \int_{0}^{t} \int \overline{I \partial_{s} u}\left(\Delta^{2} I u+F(I u)\right) d x d s .
$$


Using $I \partial_{t} u=i \Delta^{2} I u+i I F(u)$, we see that

$$
\begin{aligned}
E(I u(t))-E\left(I u_{0}\right)= & \operatorname{Re} \int_{0}^{t} \int \overline{I \partial_{s} u}(F(I u)-I F(u)) d x d s \\
= & \operatorname{Im} \int_{0}^{t} \int \overline{\Delta^{2} I u+I F(u)}(F(I u)-I F(u)) d x d s \\
= & \operatorname{Im} \int_{0}^{t} \int \overline{\Delta I u} \Delta(F(I u)-I F(u)) d x d s \\
& +\operatorname{Im} \int_{0}^{t} \int \overline{I F(u)}(F(I u)-I F(u)) d x d s
\end{aligned}
$$

We next write

$$
\begin{aligned}
\Delta(F(I u)-I F(u))= & (\Delta I u) F^{\prime}(I u)+|\nabla I u|^{2} F^{\prime \prime}(I u)-I\left(\Delta F^{\prime}(u)\right)-I\left(|\nabla u|^{2} F^{\prime \prime}(u)\right) \\
= & (\Delta I u)\left(F^{\prime}(I u)-F^{\prime}(u)\right)+|\nabla I u|^{2}\left(F^{\prime \prime}(I u)-F^{\prime \prime}(u)\right)+\nabla I u \cdot(\nabla I u-\nabla u) F^{\prime \prime}(u) \\
& +(\Delta I u) F^{\prime}(u)-I\left(\Delta u F^{\prime}(u)\right)+(I \nabla u) \cdot \nabla u F^{\prime \prime}(u)-I\left(\nabla u \cdot \nabla u F^{\prime \prime}(u)\right) .
\end{aligned}
$$

Therefore,

$$
\begin{aligned}
E(I u(t))-E\left(I u_{0}\right)= & \operatorname{Im} \int_{0}^{t} \int \overline{\Delta I u} \Delta I u\left(F^{\prime}(I u)-F^{\prime}(u)\right) d x d s \\
& +\operatorname{Im} \int_{0}^{t} \int \overline{\Delta I u}|\nabla I u|^{2}\left(F^{\prime \prime}(I u)-F^{\prime \prime}(u)\right) d x d s \\
& +\operatorname{Im} \int_{0}^{t} \int \overline{\Delta I u} \nabla I u \cdot(\nabla I u-\nabla u) F^{\prime \prime}(u) d x d s \\
& +\operatorname{Im} \int_{0}^{t} \int \overline{\Delta I u}\left[(\Delta I u) F^{\prime}(u)-I\left(\Delta u F^{\prime}(u)\right)\right] d x d s \\
& +\operatorname{Im} \int_{0}^{t} \int \overline{\Delta I u}\left[(I \nabla u) \cdot \nabla u F^{\prime \prime}(u)-I\left(\nabla u \cdot \nabla u F^{\prime \prime}(u)\right)\right] d x d s \\
& +\operatorname{Im} \int_{0}^{t} \int \overline{I F(u)}(F(I u)-I F(u)) d x d s .
\end{aligned}
$$

Let us consider (3.18). By Hölder's inequality, we estimate

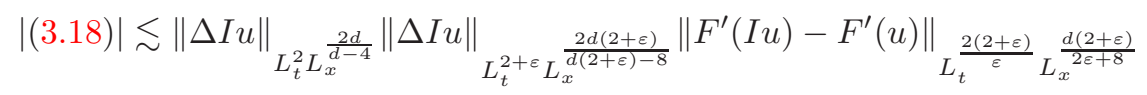

$$
\begin{aligned}
& \lesssim Z_{I}^{2}|||I u-u|(|I u|+|u|)^{\nu-2} \|_{L_{t}^{\frac{2(2+\varepsilon)}{\varepsilon}}} \frac{d(2+\varepsilon)}{L_{x}^{2 \varepsilon+8}} \\
& \lesssim Z_{I}^{2}\left\|P_{>N} u\right\|_{L_{t} \frac{2(\nu-1)(2+\varepsilon)}{\varepsilon}}^{\frac{d(\nu-1)(2+\varepsilon)}{2 \varepsilon+8}}\|u\|_{L_{t}}^{\nu-2(\nu-1)(2+\varepsilon)} \frac{\frac{2(\nu-1)(2+\varepsilon)}{\varepsilon}}{L_{x}} .
\end{aligned}
$$

Combining (3.24), (3.8) and (3.9), we get

$$
|(3.18)| \lesssim N^{\gamma_{c}-2} Z_{I}^{3}\left(\mu^{\theta} Z_{I}^{1-\theta}+Z_{I}\right)^{\nu-2} .
$$

In order to treat (3.19), we need to separate two cases $0<\nu-2<1$ and $1 \leq \nu-2$.

If $0<\nu-2<1$, then using $F^{\prime \prime}(z)=O\left(|z|^{\nu-2}\right)$, we have

$$
\left|F^{\prime \prime}(z)-F^{\prime \prime}(\zeta)\right| \lesssim|z-\zeta|^{\nu-2}, \quad \forall z, \zeta \in \mathbb{C}
$$


Moreover, there exists $k \gg 1$ so that $k(\nu-2) \geq 2$. By Hölder's inequality,

$$
\begin{aligned}
& |(3.19)| \lesssim\|\Delta I u\|_{L_{t}^{2} L_{x}^{\frac{2 d}{d-4}}}\|\nabla I u\|_{L_{t}^{\frac{4 k}{k-2}} L_{x}^{q}}^{2}\left\|F^{\prime \prime}(I u)-F^{\prime \prime}(u)\right\|_{L_{t}^{k} L_{x}^{r}} \\
& \lesssim\|\Delta I u\|_{L_{t}^{2} L_{x}^{\frac{2 d}{d-4}}}\left\||\nabla|^{1+\frac{\gamma_{c}}{2}} I u\right\|_{L_{t}^{\frac{4 k}{k-2}} L_{x}^{q^{\star}}}^{2}\left\|I u-\left.u\right|^{\nu-2}\right\|_{L_{t}^{k} L_{x}^{r}} \\
& \lesssim Z_{I}^{3}\left\|P_{>N} u\right\|_{L_{t}^{k(\nu-2)}}^{\nu-2} L_{x}^{r(\nu-2)} \\
& \lesssim Z_{I}^{3}\left\||\nabla|^{\gamma_{c}} P_{>N} u\right\|_{L_{t}^{k(\nu-2)} L_{x}^{r^{\star}}}^{\nu-2} \\
& \lesssim N^{\left(\gamma_{\mathrm{c}}-2\right)(\nu-2)} Z_{I}^{\nu+1} \text {, }
\end{aligned}
$$

where

$$
\begin{array}{rlll}
q & :=\frac{4 k d(\nu-1)}{(k d-4(k-2))(\nu-1)+8 k}, & q^{\star} \quad:=\frac{2 k d}{k d-2(k-2)}, \\
r & :=\frac{k d(\nu-1)}{4(k-1)(\nu-1)-4 k}, & r^{\star} \quad:=\frac{2 k d}{k d(\nu-2)-8} .
\end{array}
$$

Here we drop the $I$-operator and use (2.8) with the fact $1+\frac{\gamma_{c}}{2}<\gamma<2$ to have the third line. Note that $\left(\frac{4 k}{k-2}, q^{\star}\right)$ and $\left(k(\nu-2), r^{\star}\right)$ are biharmonic admissible. The last line follows from (2.7).

If $1 \leq \nu-2$, then

$$
\left|F^{\prime \prime}(z)-F^{\prime \prime}(\zeta)\right| \lesssim|z-\zeta|(|z|+|\zeta|)^{\nu-3}, \quad \forall z, \zeta \in \mathbb{C} .
$$

We estimate

$$
\begin{aligned}
|(3.19)| & \lesssim\|\Delta I u\|_{L_{t}^{2} L_{x}^{\frac{2 d}{d-4}}\|\nabla I u\|_{L_{t}^{\frac{4 k}{k-2}}}^{2} L_{x}^{q}}\left\|F^{\prime \prime}(I u)-F^{\prime \prime}(u)\right\|_{L_{t}^{k} L_{x}^{r}} \\
& \lesssim\|\Delta I u\|_{L_{t}^{2} L_{x}^{\frac{2 d}{d-4}}\left\||\nabla|^{1+\frac{\gamma c}{2}} I u\right\|_{L_{t}^{\frac{4 k}{k-2}}}^{2 q_{x}^{q^{\star}}}}\left\|F^{\prime \prime}(I u)-F^{\prime \prime}(u)\right\|_{L_{t}^{k} L_{x}^{r}} \\
& \lesssim Z_{I}^{3}\|I u-u\|_{L_{t}^{k(\nu-2)} L_{x}^{r(\nu-2)} \|}\|I u|+| u \mid\|_{L_{t}^{\nu-3}}^{\nu-2)} L_{x}^{r(\nu-2)} \\
& \lesssim Z_{I}^{3}\left\||\nabla|^{\gamma_{c}} P_{>N} u\right\|_{L_{t}^{k(\nu-2)} L_{x}^{r^{\star}}}\left\||\nabla|^{\gamma_{c}} u\right\|_{L_{t}^{k(\nu-2)} L_{x}^{r^{\star}}}^{\nu} \\
& \lesssim N^{\gamma_{c}-2} Z_{I}^{\nu+1} .
\end{aligned}
$$

Thus, collecting two cases, we obtain

$$
|(3.19)| \lesssim N^{\min \{\nu-2,1\}\left(\gamma_{c}-2\right)} Z_{I}^{\nu+1} .
$$

We next estimate

$$
\begin{aligned}
& |(3.20)| \lesssim\|\Delta I u\|_{L_{t}^{2} L_{x}^{\frac{2 d}{d-4}}}\|\nabla I u\|_{L_{t}^{\frac{4 k}{k-2}} L_{x}^{q}}\|\nabla I u-\nabla u\|_{L_{t}^{\frac{4 k}{k-2}} L_{x}^{q}}\left\|F^{\prime \prime}(u)\right\|_{L_{t}^{k} L_{x}^{r}} \\
& \lesssim\|\Delta I u\|_{L_{t}^{2} L_{x}^{\frac{2 d}{d-4}}}\|\nabla I u\|_{L_{t}^{\frac{4 k}{k-2}} L_{x}^{q}}\left\|\nabla P_{>N} u\right\|_{L_{t}^{\frac{4 k}{k-2}} L_{x}^{q}}\|u\|_{L_{t}^{k(\nu-2)} L_{x}^{r(\nu-2)}}^{\nu-2} \\
& \lesssim\|\Delta I u\|_{L_{t}^{2} \frac{2 d}{d-4}}\left\||\nabla|^{1+\frac{\gamma_{c}}{2}} I u\right\|_{L_{t}^{\frac{4 k}{k-2}} L_{x}^{q^{\star}}}\left\||\nabla|^{1+\frac{\gamma_{c}}{2}} P_{>N} u\right\|_{L_{t}^{\frac{4 k}{k-2}} L_{x}^{q^{\star}}}\left\||\nabla|^{\gamma_{c}} u\right\|_{L_{t}^{k(\nu-2)} L_{x}^{r^{*}}}^{\nu-2} \\
& \lesssim N^{\frac{\gamma_{\mathrm{c}}-2}{2}} Z_{I}^{\nu+1} \text {. }
\end{aligned}
$$

We next consider the term (3.21). Using the notation given in Lemma 3.1, we apply Corollary 2.10 with $q=\frac{2 d}{d+4}, q_{1}=\frac{2 d(2+\varepsilon)}{d(2+\varepsilon)-8}$ and $q_{2}=\frac{d(2+\varepsilon)}{2 \varepsilon+8}$ to have

$$
\left\|(\Delta I u) F^{\prime}(u)-I\left(\Delta u F^{\prime}(u)\right)\right\|_{L_{x}^{\frac{2 d}{d+4}}} \lesssim N^{-\alpha}\|\Delta I u\|_{L_{x}^{\frac{2 d(2+\varepsilon)}{d(2+\varepsilon)-8}}}\left\|\langle\nabla\rangle^{\alpha} F^{\prime}(u)\right\|_{L_{x}^{\frac{d(2+\varepsilon)}{2 \varepsilon+8}}},
$$


where $\alpha=2-\gamma+\delta$. By Hölder's inequality,

$$
\left\|(\Delta I u) F^{\prime}(u)-I\left(\Delta u F^{\prime}(u)\right)\right\|_{L_{t}^{2} L_{x}^{\frac{2 d}{d+4}}} \lesssim N^{-\alpha}\|\Delta I u\|_{L_{t}^{2+\varepsilon} L_{x}^{\frac{2 d(2+\varepsilon)}{d(2+\varepsilon)}}}\left\|\langle\nabla\rangle^{\alpha} F^{\prime}(u)\right\|_{L_{t}^{\frac{2(2+\varepsilon)}{\varepsilon}}} \frac{d(2+\varepsilon)}{L_{x}^{2 \varepsilon+8}} .
$$

We have from (3.6), (3.10) and (3.12) that

$$
\left\|\langle\nabla\rangle^{\alpha} F^{\prime}(u)\right\|_{L_{t}^{\frac{2(2+\varepsilon)}{\varepsilon}}} \frac{d(2+\varepsilon)}{L_{x}^{2 \varepsilon+8}} \lesssim\left(\mu^{\theta} Z_{I}^{1-\theta}+Z_{I}\right)^{\nu-1} .
$$

Thus

$$
\left\|(\Delta I u) F^{\prime}(u)-I\left(\Delta u F^{\prime}(u)\right)\right\|_{L_{t}^{2} L_{x}^{\frac{2 d}{d+4}}} \lesssim N^{-\alpha} Z_{I}\left(\mu^{\theta} Z_{I}^{1-\theta}+Z_{I}\right)^{\nu-1}
$$

and

$$
|(3.21)| \lesssim N^{-(2-\gamma+\delta)} Z_{I}^{2}\left(\mu^{\theta} Z_{I}^{1-\theta}+Z_{I}\right)^{\nu-1}
$$

Similarly,

$$
|(3.22)| \lesssim\|\Delta I u\|_{L_{t}^{2} L_{x}^{\frac{2 d}{d-4}}}\left\|(I \nabla u) \cdot \nabla u F^{\prime \prime}(u)-I\left(\nabla u \cdot \nabla u F^{\prime \prime}(u)\right)\right\|_{L_{t}^{2} L_{x}^{\frac{2 d}{d+4}}} \cdot
$$

We next apply Lemma 2.9 with $q=\frac{2 d}{d+4}, q_{1}=\frac{4 k d(\nu-1)}{(k d-4(k-2))(\nu-1)+8 k}$ and $q_{2}=\frac{4 k d(\nu-1)}{(k d+12 k-8)(\nu-1)-8 k}$ to have

$$
\begin{aligned}
& \left\|(I \nabla u) \cdot \nabla u F^{\prime \prime}(u)-I\left(\nabla u \cdot \nabla u F^{\prime \prime}(u)\right)\right\|_{L_{x}^{\frac{2 d}{d+4}}} \lesssim N^{-\alpha}\|I \nabla u\|_{L_{x}^{\frac{1(k d-4(k-2))(\nu-1)+8 k}{(k)}}}
\end{aligned}
$$

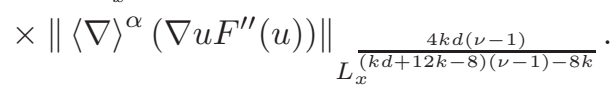

Using the notation (3.26), the fractional chain rule implies

$$
\left\|\langle\nabla\rangle^{\alpha}\left(\nabla u F^{\prime \prime}(u)\right)\right\|_{L_{x}^{(k d+12 k-8)(\nu-1)-8 k}} \lesssim\left\|\langle\nabla\rangle^{1+\alpha} u\right\|_{L_{x}^{q}}\left\|F^{\prime \prime}(u)\right\|_{L_{x}^{r}}+\|\nabla u\|_{L_{x}^{q}}\left\|\langle\nabla\rangle^{\alpha} F^{\prime \prime}(u)\right\|_{L_{x}^{r}} .
$$

The Hölder inequality then gives

$$
\begin{aligned}
\|(I \nabla u) \cdot \nabla u F^{\prime \prime}(u)- & I\left(\nabla u \cdot \nabla u F^{\prime \prime}(u)\right)\left\|_{L_{t}^{2} L_{x}^{\frac{2 d}{d+4}}} \lesssim N^{-\alpha}\right\| I \nabla u \|_{L_{t}^{\frac{4 k}{k-2}} L_{x}^{q}} \\
& \times\left(\left\|\langle\nabla\rangle^{1+\alpha} u\right\|_{L_{t}^{\frac{4 k}{k-2}} L_{x}^{q}}\left\|F^{\prime \prime}(u)\right\|_{L_{t}^{k} L_{x}^{r}}+\|\nabla u\|_{L_{t}^{\frac{4 k}{k-2}} L_{x}^{q}}\left\|\langle\nabla\rangle^{\alpha} F^{\prime \prime}(u)\right\|_{L_{t}^{k} L_{x}^{r}}\right) .
\end{aligned}
$$

By the Sobolev embedding (dropping the $I$-operator if necessary) and (2.8), we have

$$
\begin{aligned}
& \|I \nabla u\|_{L_{t}^{\frac{4 k}{k-2}} L_{x}^{q}},\|\nabla u\|_{L_{t}^{\frac{4 k}{k-2}} L_{x}^{q}} \lesssim\left\||\nabla|^{1+\frac{\gamma_{c}}{2}} u\right\|_{L_{t}^{\frac{4 k}{k-2}} L_{L^{q^{\star}}}} \lesssim Z_{I}, \\
& \left\|\langle\nabla\rangle^{1+\alpha} u\right\|_{L_{t}^{\frac{4 k}{k-2}} L_{x}^{q}} \lesssim\left\|\langle\nabla\rangle^{1+\alpha+\frac{\gamma_{c}}{2}} u\right\|_{L_{t}^{\frac{4 k}{k-2}} L_{x}^{q^{\star}}} \lesssim Z_{I} .
\end{aligned}
$$

Note that by our assumptions on $\delta, 1+\alpha+\frac{\gamma_{c}}{2}=3-\gamma+\delta+\frac{\gamma_{c}}{2}<\gamma$. We also have

$$
\left\|F^{\prime \prime}(u)\right\|_{L_{t}^{k} L_{x}^{r}} \lesssim\|u\|_{L_{t}^{k(\nu-2)} L_{x}^{r(\nu-2)}}^{\nu-2} \lesssim\left\||\nabla|^{\gamma_{c}} u\right\|_{L_{t}^{k(\nu-2)} L_{x}^{r^{\star}}}^{\nu-2} \lesssim Z_{I}^{\nu-2} .
$$

It remains to treat $\left\|\langle\nabla\rangle^{\alpha} F^{\prime \prime}(u)\right\|_{L_{t}^{k} L_{x}^{r}}$. Using (3.32), we only need to bound $\left\||\nabla|^{\alpha} F^{\prime \prime}(u)\right\|_{L_{t}^{k} L_{x}^{r}}$. To do so, we separate two cases: $1 \leq \nu-2$ and $0<\nu-2<1$. 
If $1 \leq \nu-2$, then we apply Lemma 2.1 for $q=r, q_{1}=\frac{r(\nu-2)}{\nu-3}, q_{2}=r(\nu-2)$ and use Hölder's inequality to have

$$
\begin{aligned}
& \left\||\nabla|^{\alpha} F^{\prime \prime}(u)\right\|_{L_{t}^{k} L_{x}^{r}} \lesssim\left\|O\left(|u|^{\nu-3}\right)\right\|_{L_{t}^{\frac{k(\nu-2)}{\nu-3}}} L_{x}^{\frac{r(\nu-2)}{\nu-3}}\left\||\nabla|^{\alpha} u\right\|_{L_{t}^{k(\nu-2)} L_{x}^{r(\nu-2)}} \\
& \lesssim\|u\|_{L_{t}^{k(\nu-2)} L_{x}^{r(\nu-2)}}^{\nu-3}\left\||\nabla|^{\alpha} u\right\|_{L_{t}^{k(\nu-2)} L_{x}^{r(\nu-2)}} \\
& \lesssim\left\||\nabla|_{c}^{\gamma} u\right\|_{L_{t}^{k(\nu-2)} L_{x}^{r^{\star}}}^{\nu-3}\left\||\nabla|^{\alpha+\gamma_{c}} u\right\|_{L_{t}^{k(\nu-2)} L_{x}^{r^{\star}}} \\
& \lesssim Z_{I}^{\nu-2} \text {. }
\end{aligned}
$$

Here by our assumptions, $\alpha+\gamma_{\mathrm{c}}<\gamma$ which allows us to use (2.8) to get the last estimate.

If $0<\nu-2<1$, then we use Lemma 2.2 with $\beta=\nu-2, \alpha=2-\gamma+\delta, q=r$ and $q_{1}, q_{2}$ satisfying

$$
\left(\nu-2-\frac{\alpha}{\rho}\right) q_{1}=\frac{\alpha}{\rho} q_{2}=r(\nu-2),
$$

and $\frac{\alpha}{\nu-2}<\rho<1$ to be chosen later. With these choices, we have

$$
\left(1-\frac{\alpha}{\beta \rho}\right) q_{1}=r>1
$$

Then,

$$
\left\||\nabla|^{\alpha} F^{\prime \prime}(u)\right\|_{L_{x}^{r}} \lesssim\left\||u|^{\nu-2-\frac{\alpha}{\rho}}\right\|_{L_{x}^{q_{1}}}\left\||\nabla|^{\rho} u\right\|_{L_{x}^{\frac{\alpha}{\rho} q_{2}}}^{\frac{\alpha}{\rho}} \lesssim\|u\|_{L_{x}^{\left(\nu-2-\frac{\alpha}{\rho}\right) q_{1}}}^{\nu-2-\frac{\alpha}{\rho}}\left\||\nabla|^{\rho} u\right\|_{L_{x}^{\frac{\alpha}{\rho}}}^{\frac{\alpha}{\rho}}
$$

By Hölder's inequality,

$$
\begin{aligned}
& \left\||\nabla|^{\alpha} F^{\prime \prime}(u)\right\|_{L_{t}^{k} L_{x}^{r}} \lesssim\|u\|_{L_{t}^{\left(\nu-2-\frac{\alpha}{\rho}\right) p_{1}} L_{x}^{\left(\nu-2-\frac{\alpha}{\rho}\right) q_{1}}}^{\nu}\left\||\nabla|^{\rho} u\right\|_{L_{t}^{\frac{\rho}{\rho} p_{2}}}^{\frac{\alpha}{\rho}} L_{x}^{\frac{\alpha}{\rho} q_{2}} \\
& \lesssim\|u\|_{L_{t}^{k(\nu-2)} L_{x}^{r(\nu-2)}}^{\nu-2-\frac{\alpha}{\rho}}\left\||\nabla|^{\rho} u\right\|_{L_{t}^{k(\nu-2)} L_{x}^{r(\nu-2)}},
\end{aligned}
$$

provided that

$$
\left(\nu-2-\frac{\alpha}{\rho}\right) p_{1}=\frac{\alpha}{\rho} p_{2}=k(\nu-2) .
$$

The Sobolev imbedding then gives

$$
\left\||\nabla|^{\alpha} F^{\prime \prime}(u)\right\|_{L_{t}^{k} L_{x}^{r}} \lesssim\left\||\nabla|^{\gamma_{c}} u\right\|_{L_{t}^{k(\nu-2)} L_{x}^{r^{\star}}}^{\nu-2-\frac{\alpha}{\alpha}}\left\||\nabla|^{\rho+\gamma_{c}} u\right\|_{L_{t}^{k(\nu-2)} L_{x}^{r^{\star}}} \lesssim Z_{I}^{\nu-2} .
$$

Here we use (2.8) together with $\rho+\gamma_{\mathrm{c}}<\gamma$ to get the last estimate. Note that

$$
\frac{\alpha}{\nu-2}+\gamma_{\mathrm{c}}<\rho+\gamma_{\mathrm{c}}
$$

If we want $\rho+\gamma_{\mathrm{c}}<\gamma$ for an appropriate value of $\rho$, we need $\frac{\alpha}{\nu-2}+\gamma_{\mathrm{c}}<\gamma$. This implies $\gamma>\frac{2}{\nu-1}+\frac{\nu-2}{\nu-1} \gamma_{\mathrm{c}}$ and $\delta<(\nu-1) \gamma-2-(\nu-2) \gamma_{\mathrm{c}}$. Collecting 2 cases, we show

$$
\left\||\nabla|{ }^{\alpha} F^{\prime \prime}(u)\right\|_{L_{t}^{k} L_{x}^{r}} \lesssim Z_{I}^{\nu-2} .
$$

By $(3.30),(3.31),(3.32)$ and (3.33),

$$
\left\|(I \nabla u) \cdot \nabla u F^{\prime \prime}(u)-I\left(\nabla u \cdot \nabla u F^{\prime \prime}(u)\right)\right\|_{L_{t}^{2} L_{x}^{\frac{2 d}{d+4}}} \lesssim N^{-\alpha} Z_{I}^{\nu} .
$$

Thus,

$$
|(3.22)| \lesssim N^{-(2-\gamma+\delta)} Z_{I}^{\nu+1} .
$$


Finally, we consider (3.23). We bound

$$
\begin{aligned}
|(3.23)| & \lesssim\left\||\nabla|^{-1} I F(u)\right\|_{L_{t}^{2} L_{x}^{\frac{2 d}{d-2}}}\|\nabla(F(I u)-I F(u))\|_{L_{t}^{2} L_{x}^{\frac{2 d}{d+2}}} \\
& \lesssim\|\nabla I F(u)\|_{L_{t}^{2} L_{x}^{\frac{2 d}{d+2}}}\|\nabla(F(I u)-I F(u))\|_{L_{t}^{2} L_{x}^{\frac{2 d}{d+2}}}
\end{aligned}
$$

By (3.2),

$$
\|\nabla I F(u)\|_{L_{t}^{2} L_{x}^{\frac{2 d}{d+2}}} \lesssim N^{-(2-\gamma+\delta)} Z_{I}^{\nu}+\mu^{(\nu-1) \theta} Z_{I}^{1+(\nu-1)(1-\theta)} .
$$

By the triangle inequality,

$$
\|\nabla(F(I u)-I F(u))\|_{L_{t}^{2} L_{x}^{\frac{2 d}{d+2}}} \lesssim\left\|(\nabla I u)\left(F^{\prime}(I u)-F^{\prime}(u)\right)\right\|_{L_{t}^{2} L_{x}^{\frac{2 d}{d+2}}}+\left\|(\nabla I u) F^{\prime}(u)-\nabla I F(u)\right\|_{L_{t}^{2} L_{x}^{\frac{2 d}{d+2}}} .
$$

We firstly use Hölder's inequality and estimate as in (3.24) to get

$$
\begin{aligned}
& \left\|(\nabla I u)\left(F^{\prime}(I u)-F^{\prime}(u)\right)\right\|_{L_{t}^{2} L_{x}^{\frac{2 d}{d+2}}} \lesssim\|\nabla I u\|_{L_{t}^{2+\varepsilon} L_{x} \frac{2 d(2+\varepsilon)}{d(2+\varepsilon)-12}}\left\|F^{\prime}(I u)-F^{\prime}(u)\right\|_{L_{t}^{\frac{2(2+\varepsilon)}{\varepsilon}}} L_{x}^{\frac{d(2+\varepsilon)}{2 \varepsilon+8}} \\
& \lesssim\|\Delta I u\|_{L_{t}^{2+\varepsilon} L_{x}^{\frac{2 d(2+\varepsilon)}{d(2+\varepsilon)-8}}}\left\|F^{\prime}(I u)-F^{\prime}(u)\right\|_{L_{t}^{\frac{2(2+\varepsilon)}{\varepsilon}}} \frac{d(2+\varepsilon)}{L_{x}^{2 \varepsilon+8}} \\
& \lesssim N^{\gamma_{c}-2} Z_{I}^{2}\left(\mu^{\theta} Z_{I}^{1-\theta}+Z_{I}\right)^{\nu-2} \text {. }
\end{aligned}
$$

By (3.1),

$$
\left\|(\nabla I u) F^{\prime}(u)-\nabla I F(u)\right\|_{L_{t}^{2} L_{x}^{\frac{2 d}{d+2}}} \lesssim N^{-(2-\gamma+\delta)} Z_{I}\left(\mu^{\theta} Z_{I}^{1-\theta}+Z_{I}\right)^{\nu-1} .
$$

Combining (3.35) - (3.38), we get

$$
|(3.23)| \lesssim N^{-(2-\gamma+\delta)} Z_{I}^{2}\left(\mu^{\theta} Z_{I}^{1-\theta}+Z_{I}\right)^{2(\nu-1)}
$$

Collecting (3.25), (3.27), (3.28), (3.29), (3.34), (3.39) and using (3.17), we prove (3.14). Note that our assumptions on $\delta$ implies

$$
2-\gamma+\delta<\min \left\{\gamma-1-\frac{\gamma_{\mathrm{c}}}{2}, \nu-2,(\nu-2)\left(\gamma-\gamma_{\mathrm{c}}\right)\right\}<\min \left\{\frac{2-\gamma_{\mathrm{c}}}{2},(\nu-2)\left(2-\gamma_{\mathrm{c}}\right)\right\} .
$$

The proof is complete.

\section{Global Well-Posedness and scattering}

In this section, we shall give the proof of the global existence and scattering given in Theorem 1.1 .

Global well-posedness. By the density argument, the proof of global well-posedness will be reduced to the following.

Proposition 4.1. Let $5 \leq d \leq 11$ and $\gamma(d, \nu)<\gamma<2$ with $\gamma(d, \nu)$ be as in (1.5). Suppose that $u$ is a global solution to (NL4S) with initial data $u_{0} \in C_{0}^{\infty}\left(\mathbb{R}^{d}\right)$. Then,

$$
\begin{aligned}
\|u\|_{M^{\sigma}(\mathbb{R})} & \leq C\left(\left\|u_{0}\right\|_{H_{x}^{\gamma}}\right), \\
\|u\|_{L_{t}^{\infty}\left(\mathbb{R}, H_{x}^{\gamma}\right)} & \leq C\left(\left\|u_{0}\right\|_{H_{x}^{\gamma}}\right),
\end{aligned}
$$

where $\|\cdot\|_{M^{\sigma}}$ is given in (2.15). 
Proof. The proof of this result is based on the almost conservation law given in Proposition 3.3. To do so, we need the modified energy of initial data is small. Since $E\left(I u_{0}\right)$ is not necessarily small, we use the scaling (1.2) to make $E\left(\operatorname{Iu}_{\lambda}(0)\right)$ small. We have

$$
E\left(I u_{\lambda}(0)\right)=\frac{1}{2}\left\|I u_{\lambda}(0)\right\|_{\dot{H}_{x}^{2}}^{2}+\frac{1}{\nu+1}\left\|I u_{\lambda}(0)\right\|_{L_{x}^{\nu+1}}^{\nu+1} .
$$

By (2.10),

$$
\left\|I u_{\lambda}(0)\right\|_{\dot{H}_{x}^{2}} \lesssim N^{2-\gamma}\left\|u_{\lambda}(0)\right\|_{\dot{H}_{x}^{\gamma}}=N^{2-\gamma} \lambda^{\gamma_{c}-\gamma}\left\|u_{0}\right\|_{\dot{H}_{x}^{\gamma}}
$$

By choosing

$$
\lambda \approx N^{\frac{2-\gamma}{\gamma-\gamma_{c}}}
$$

we have $\left\|I u_{\lambda}(0)\right\|_{\dot{H}_{x}^{2}} \leq \frac{1}{8}$. We next bound $\left\|I u_{\lambda}(0)\right\|_{L_{x}^{\nu+1}}$. Note that we can easily estimate this norm by the Sobolev embedding,

$$
\left\|I u_{\lambda}(0)\right\|_{L_{x}^{\nu+1}} \lesssim\left\|u_{\lambda}(0)\right\|_{L_{x}^{\nu+1}}=\lambda^{\frac{d}{\nu+1}-\frac{4}{\nu-1}}\left\|u_{0}\right\|_{L_{x}^{\nu+1}} \lesssim \lambda^{\frac{(d-4)(\nu-1)-8}{(\nu+1)(\nu-1)}}\left\|u_{0}\right\|_{H_{x}^{\gamma}}
$$

provided that $\gamma \geq \frac{d(\nu-1)}{2(\nu+1)}$. In order to remove this unexpected condition on $\gamma$, we use the technique of [CKSTT04] (see also [MWZ15]). We firstly separate the frequency space into the domains

$$
\Omega_{1}:=\left\{\xi \in \mathbb{R}^{d},|\xi| \lesssim \frac{1}{\lambda}\right\}, \quad \Omega_{2}:=\left\{\xi \in \mathbb{R}^{d}, \frac{1}{\lambda} \lesssim|\xi| \lesssim N\right\}, \quad \Omega_{3}:=\left\{\xi \in \mathbb{R}^{d},|\xi| \gtrsim N\right\},
$$

and then write

$$
\left[u_{\lambda}(0)\right\}^{\Upsilon}(\xi)=\left(\chi_{1}(\xi)+\chi_{2}(\xi)+\chi_{3}(\xi)\right)\left[u_{\lambda}(0) \varsigma^{\Upsilon}(\xi),\right.
$$

for non-negative smooth functions $\chi_{j}$ supported in $\Omega_{j}, j=1,2,3$ respectively and satisfying $\sum \chi_{j}(\xi)=1$. Thus

$$
I u_{\lambda}(0)=\chi_{1}(D) I u_{\lambda}(0)+\chi_{2}(D) I u_{\lambda}(0)+\chi_{3}(D) I u_{\lambda}(0) .
$$

We now use the Sobolev embedding to have

$$
\left\|\chi_{1}(D) I u_{\lambda}(0)\right\|_{L_{x}^{\nu+1}} \lesssim\left\||\nabla|^{\frac{d(\nu-1)}{2(\nu+1)}} \chi_{1}(D) I u_{\lambda}(0)\right\|_{L_{x}^{2}} \lesssim\left\||\nabla|^{\frac{d(\nu-1)}{2(\nu+1)}} \chi_{1}(D) I\right\|_{L_{x}^{2} \rightarrow L_{x}^{2}}\left\|u_{\lambda}(0)\right\|_{L_{x}^{2}} .
$$

Thanks to the support of $\chi_{1}$, the functional calculus gives

$$
\left\||\nabla|^{\frac{d(\nu-1)}{2(\nu+1)}} \chi_{1}(D) I\right\|_{L_{x}^{2} \rightarrow L_{x}^{2}} \lesssim\left\||\xi|^{\frac{d(\nu-1)}{2(\nu+1)}-\alpha}|\xi|^{\alpha} \chi_{1}(\xi)\right\|_{L_{\xi}^{\infty}} \lesssim \lambda^{\alpha-\frac{d(\nu-1)}{2(\nu+1)}},
$$

provided $0<\alpha<\frac{d(\nu-1)}{2(\nu+1)}$. Similarly,

$$
\left\|\chi_{3}(D) I u_{\lambda}(0)\right\|_{L_{x}^{\nu+1}} \lesssim\left\||\nabla|^{\frac{d(\nu-1)}{2(\nu+1)}} \chi_{3}(D) I u_{\lambda}(0)\right\|_{L_{x}^{2}} \lesssim\left\||\nabla|^{\frac{d(\nu-1)}{2(\nu+1)}-\gamma} \chi_{3}(D) I\right\|_{L_{x}^{2} \rightarrow L_{x}^{2}}\left\|u_{\lambda}(0)\right\|_{\dot{H}_{x}^{\gamma}} .
$$

A direct computation shows

$$
\left\|u_{\lambda}(0)\right\|_{\dot{H}_{x}^{\gamma}}=\lambda^{-\gamma}\left\|u_{0}\right\|_{\dot{H}_{x}^{\gamma}}
$$

Using the support of $\chi_{3}$, the functional calculus again gives

$$
\left\||\nabla|^{\frac{d(\nu-1)}{2(\nu+1)}-\gamma} \chi_{3}(D) I\right\|_{L_{x}^{2} \rightarrow L_{x}^{2}} \lesssim\left\||\xi|^{\frac{d(\nu-1)}{2(\nu+1)}-\gamma} \chi_{3}(\xi)\left(N|\xi|^{-1}\right)^{2-\gamma}\right\|_{L_{\xi}^{\infty}} \lesssim N^{\frac{d(\nu-1)}{2(\nu+1)}-\gamma} .
$$

To obtain this bound, we split into two cases.

When $\frac{d(\nu-1)}{2(\nu+1)} \geq \gamma$, we simply bound

$$
\left\||\xi|^{\frac{d(\nu-1)}{2(\nu+1)}-\gamma} \chi_{3}(\xi)\left(N|\xi|^{-1}\right)^{2-\gamma}\right\|_{L_{\xi}^{\infty}} \lesssim 1 \lesssim N^{\frac{d(\nu-1)}{2(\nu+1)}-\gamma} .
$$


When $\gamma>\frac{d(\nu-1)}{2(\nu+1)}$, we write

$$
\begin{aligned}
\left\||\xi|^{\frac{d(\nu-1)}{2(\nu+1)}-\gamma} \chi_{3}(\xi)\left(N|\xi|^{-1}\right)^{2-\gamma}\right\|_{L_{\xi}^{\infty}} & =N^{\frac{d(\nu-1)}{2(\nu+1)}-\gamma}\left\|\left(N|\xi|^{-1}\right)^{\gamma-\frac{d(\nu-1)}{2(\nu+1)}} \chi_{3}(\xi)\left(N|\xi|^{-1}\right)^{2-\gamma}\right\|_{L_{\xi}^{\infty}} \\
& \lesssim N^{\frac{d(\nu-1)}{2(\nu+1)}-\gamma} .
\end{aligned}
$$

Combining (4.7) and (4.8), we get

$$
\left\|\chi_{3}(D) I u_{\lambda}(0)\right\|_{L_{x}^{\nu+1}} \lesssim N^{\frac{d(\nu-1)}{2(\nu+1)}-\gamma} \lambda^{-\gamma}\left\|u_{0}\right\|_{\dot{H}_{x}^{\gamma}}
$$

We treat the intermediate case as

$$
\left\|\chi_{2}(D) I u_{\lambda}(0)\right\|_{L_{x}^{\nu+1}} \lesssim\left\||\nabla|^{\frac{d(\nu-1)}{2(\nu+1)}-\gamma} \chi_{2}(D) I\right\|_{L_{x}^{2} \rightarrow L_{x}^{2}}\left\|u_{\lambda}(0)\right\|_{\dot{H}_{x}^{\gamma}} .
$$

We have

$$
\left\||\nabla|^{\frac{d(\nu-1)}{2(\nu+1)}-\gamma} \chi_{2}(D) I\right\|_{L_{x}^{2} \rightarrow L_{x}^{2}} \lesssim\left\|\left.\xi\right|^{\frac{d(\nu-1)}{2(\nu+1)}-\gamma} \chi_{2}(\xi)\right\|_{L_{\xi}^{\infty}} .
$$

When $\frac{d(\nu-1)}{2(\nu+1)} \geq \gamma$, we bound

$$
\left\|\left.\xi\right|^{\frac{d(\nu-1)}{2(\nu+1)}-\gamma} \chi_{2}(\xi)\right\|_{L_{\xi}^{\infty}} \lesssim N^{\frac{d(\nu-1)}{2(\nu+1)}-\gamma} .
$$

When $\gamma>\frac{d(\nu-1)}{2(\nu+1)}$, we write

$$
\left\|\left.\xi\right|^{\frac{d(\nu-1)}{2(\nu+1)}-\gamma} \chi_{2}(\xi)\right\|_{L_{\xi}^{\infty}}=\left\|\left.\xi\right|^{\frac{d(\nu-1)}{2(\nu+1)}-\gamma-\beta}|\xi|^{\beta} \chi_{2}(\xi)\right\|_{L_{\xi}^{\infty}} \lesssim \lambda^{\beta+\gamma-\frac{d(\nu-1)}{2(\nu+1)}},
$$

provided $\frac{d(\nu-1)}{2(\nu+1)}-\gamma<\beta<\frac{d(\nu-1)}{2(\nu+1)}$. These estimates together with (4.7) yield

$$
\left\|\chi_{2}(D) I u_{\lambda}(0)\right\|_{L_{x}^{\nu+1}} \lesssim \begin{cases}N^{\frac{d(\nu-1)}{2(\nu+1)}-\gamma} \lambda^{-\gamma}\left\|u_{0}\right\|_{\dot{H}_{x}^{\gamma}} & \text { if } \frac{d(\nu-1)}{2(\nu+1)} \geq \gamma \\ \lambda^{\beta-\frac{d(\nu-1)}{2(\nu+1)}}\left\|u_{0}\right\|_{\dot{H}_{x}^{\gamma}} & \text { if } \gamma>\frac{d(\nu-1)}{2(\nu+1)}\end{cases}
$$

Collecting (4.6), (4.9), (4.10) and use (4.5), we obtain

$$
\left\|I u_{\lambda}(0)\right\|_{L_{x}^{\nu+1}} \lesssim\left(\lambda^{\alpha-\frac{d(\nu-1)}{2(\nu+1)}}+\lambda^{\beta-\frac{d(\nu-1)}{2(\nu+1)}}+\lambda^{\frac{((d-4)(\nu-1)-8) \gamma}{2(2-\gamma)(\nu+1)}}\right)\left\|u_{0}\right\|_{H_{x}^{\gamma}},
$$

for some $0<\alpha<\frac{d(\nu-1)}{2(\nu+1)}$ and $\frac{d(\nu-1)}{2(\nu+1)}-\gamma<\beta<\frac{d(\nu-1)}{2(\nu+1)}$. Therefore, it follows from (4.4), (4.5) and (4.11) by taking $\lambda$ sufficiently large depending on $\left\|u_{0}\right\|_{H_{x}^{\gamma}}$ and $N$ (which will be chosen later and depend only on $\left\|u_{0}\right\|_{H_{x}^{\gamma}}$ ) that

$$
E\left(I u_{\lambda}(0)\right) \leq \frac{1}{4}
$$

We now show that there exists an absolute constant $C$ such that

$$
\left\|u_{\lambda}\right\|_{M^{\sigma}(\mathbb{R})} \leq C \lambda^{\gamma_{c}+\frac{\sigma(4-d) \gamma_{c}}{2(d-5+4 \sigma)}} .
$$

By undoing the scaling, using the fact that

$$
\left\|u_{\lambda}\right\|_{M^{\sigma}(\mathbb{R})}=\lambda^{\gamma_{c}+\frac{\sigma(4-d)}{d-5+4 \sigma}}\|u\|_{M^{\sigma}(\mathbb{R})},
$$

we get (4.1). We shall use the bootstrap argument to show (4.12). By time reversal symmetry, it suffices to treat the positive time only. To do so, we define

$$
\Omega_{1}:=\left\{t \in[0, \infty) \mid\left\|u_{\lambda}\right\|_{M^{\sigma}([0, t])} \leq C \lambda^{\gamma_{\mathrm{c}}+\frac{\sigma(4-d) \gamma_{\mathrm{c}}}{2(d-5+4 \sigma)}}\right\} .
$$

We want to show $\Omega_{1}=[0, \infty)$. Let

$$
\Omega_{2}:=\left\{t \in[0, \infty) \mid\left\|u_{\lambda}\right\|_{M^{\sigma}([0, t])} \leq 2 C \lambda^{\gamma_{c}+\frac{\sigma(4-d) \gamma_{c}}{2(d-5+4 \sigma)}}\right\}
$$

In order to run the bootstrap argument successfully, we need to verify four things: 
1) $\Omega_{1} \neq \emptyset$. This is obvious as $0 \in \Omega_{1}$.

2) $\Omega_{1}$ is closed. This follows from Fatou's Lemma.

3) $\Omega_{2} \subset \Omega_{1}$.

4) If $T \in \Omega_{1}$, then there exists $\delta>0$ such that $[T, T+\delta) \subset \Omega_{2}$. This is a consequence of the local well-posedness and 3 ).

It remains to prove 3). Fix $T \in \Omega_{2}$, we will show that $T \in \Omega_{1}$. We firstly use the interaction Morawetz inequality (2.14) and the mass conservation to have

$$
\begin{aligned}
\left\|u_{\lambda}\right\|_{M^{\sigma}([0, T])} & \lesssim\left(\left\|u_{\lambda}(0)\right\|_{L_{x}^{2}}\left\|u_{\lambda}\right\|_{L_{t}^{\infty}\left([0, T], \dot{H}_{x}^{\frac{1}{2}}\right)}\right)^{\frac{2 \sigma}{d-5+4 \sigma}}\left\|u_{\lambda}\right\|_{L_{t}^{\infty}\left([0, T], \dot{H}_{x}^{\sigma}\right)}^{\frac{d-5}{d-4 \sigma}} \\
& \lesssim C\left(\left\|u_{0}\right\|_{L_{x}^{2}}\right) \lambda^{\frac{2 \sigma \gamma_{c}}{d-5+4 \sigma}}\left\|u_{\lambda}\right\|_{L_{t}^{\infty}\left([0, T], \dot{H}_{x}^{\frac{1}{2}}\right)}^{\frac{2 \sigma}{d-5+4 \sigma}}\left\|u_{\lambda}\right\|_{L_{t}^{\infty}\left([0, T], \dot{H}_{x}^{\sigma}\right)}^{\frac{d-5}{d-5+4 \sigma}} .
\end{aligned}
$$

We now decompose

$$
u_{\lambda}(t):=P_{\leq N} u_{\lambda}(t)+P_{>N} u_{\lambda}(t)
$$

to estimate the second and the third factor in the right hand side of (4.13). For the low frequency part, we interpolate between the $L_{x}^{2}$-norm and $\dot{H}_{x}^{2}$-norm to have

$$
\begin{aligned}
&\left\|P_{\leq N} u_{\lambda}(t)\right\|_{\dot{H}_{x}^{\frac{1}{2}}} \lesssim\left\|P_{\leq N} u_{\lambda}(t)\right\|_{L_{x}^{2}}^{\frac{3}{4}}\left\|P_{\leq N} u_{\lambda}(t)\right\|_{\dot{H}_{x}^{2}}^{\frac{1}{4}} \lesssim C\left(\left\|u_{0}\right\|_{L_{x}^{2}}\right) \lambda^{\frac{3 \gamma_{c}}{4}}\left\|I u_{\lambda}(t)\right\|_{\dot{H}_{x}^{2}}^{\frac{1}{4}}, \\
&\left\|P_{\leq N} u_{\lambda}(t)\right\|_{\dot{H}_{x}^{\sigma}} \lesssim\left\|P_{\leq N} u_{\lambda}(t)\right\|_{L_{x}^{2}}^{1-\frac{\sigma}{2}}\left\|P_{\leq N} u_{\lambda}(t)\right\|_{\dot{H}_{x}^{2}}^{\frac{\sigma}{2}} \lesssim C\left(\left\|u_{0}\right\|_{L_{x}^{2}}\right) \lambda^{\frac{\gamma_{c}(2-\sigma)}{2}}\left\|I u_{\lambda}(t)\right\|_{\dot{H}_{x}^{2}}^{\frac{\sigma}{2}} .
\end{aligned}
$$

Note that the $I$-operator is the identity on low frequency $|\xi| \leq N$. For high frequency part, we interpolate between the $L_{x}^{2}$-norm and $\dot{H}_{x}^{\gamma}$-norm and use (2.7) to have

$$
\begin{aligned}
\left\|P_{>N} u_{\lambda}(t)\right\|_{\dot{H}_{x}^{\frac{1}{2}}} & \lesssim\left\|P_{>N} u_{\lambda}(t)\right\|_{L_{x}^{2}}^{1-\frac{1}{2 \gamma}}\left\|P_{>N} u_{\lambda}(t)\right\|_{\dot{H}_{x}^{\gamma}}^{\frac{1}{2 \gamma}} \\
& \lesssim C\left(\left\|u_{0}\right\|_{L_{x}^{2}}\right) \lambda^{\gamma_{c}\left(1-\frac{1}{2 \gamma}\right)} N^{\frac{\gamma-2}{2 \gamma}}\left\|I u_{\lambda}(t)\right\|_{\dot{H}_{x}^{2}}^{\frac{1}{2 \gamma}} \\
& \lesssim C\left(\left\|u_{0}\right\|_{L_{x}^{2}}\right) \lambda^{\frac{3 \gamma_{c}}{4}}\left\|I u_{\lambda}(t)\right\|_{\dot{H}_{x}^{2}}^{\frac{1}{2 \gamma}}, \\
\left\|P_{>N} u_{\lambda}(t)\right\|_{\dot{H}_{x}^{\sigma}} & \lesssim\left\|P_{>N} u_{\lambda}(t)\right\|_{L_{x}^{2}}^{1-\frac{\sigma}{\gamma}}\left\|P_{>N} u_{\lambda}(t)\right\|_{\dot{H}_{x}^{2}}^{\frac{\sigma}{\gamma}} \\
& \lesssim C\left(\left\|u_{0}\right\|_{L_{x}^{2}}\right) \lambda^{\gamma_{c}\left(1-\frac{\sigma}{\gamma}\right)} N^{\frac{\sigma(\gamma-2)}{\gamma}}\left\|I u_{\lambda}(t)\right\|_{\dot{H}_{x}^{2}}^{\frac{\sigma}{\gamma}} \\
& \lesssim C\left(\left\|u_{0}\right\|_{L_{x}^{2}}\right) \lambda^{\frac{\gamma_{c}(2-\sigma)}{2}}\left\|I u_{\lambda}(t)\right\|_{\dot{H}_{x}^{2}}^{\frac{\sigma}{\gamma}} .
\end{aligned}
$$

Here we use the fact $0<\gamma<2$ to get (4.16) and (4.17). Collecting (4.13) through (4.17), we get

$$
\begin{aligned}
& \left\|u_{\lambda}\right\|_{M^{\sigma}([0, T])} \lesssim C\left(\left\|u_{0}\right\|_{L_{x}^{2}}\right) \lambda^{\gamma_{c}+\frac{\sigma(4-d) \gamma_{c}}{2(d-5+4 \sigma)}} \sup _{[0, T]}\left(\left\|I u_{\lambda}(t)\right\|_{\dot{H}_{x}^{2}}^{\frac{1}{4}}+\left\|I u_{\lambda}(t)\right\|_{\dot{H}_{x}^{2}}^{\frac{1}{2 \gamma}}\right)^{\frac{2 \sigma}{d-5+4 \sigma}} \\
& \times \sup _{[0, T]}\left(\left\|I u_{\lambda}(t)\right\|_{\dot{H}_{x}^{2}}^{\frac{\sigma}{2}}+\left\|I u_{\lambda}(t)\right\|_{\dot{H}_{x}^{2}}^{\frac{\sigma}{\gamma}}\right)^{\frac{d-5}{d-5+4 \sigma}} .
\end{aligned}
$$

Thus, by taking $C$ sufficiently large depending on $\left\|u_{0}\right\|_{L_{x}^{2}}$, we get $T \in \Omega_{1}$, provided that

$$
\sup _{[0, T]}\left\|I u_{\lambda}(t)\right\|_{\dot{H}_{x}^{2}} \lesssim 1
$$

We will prove that (4.19) holds for $T \in \Omega_{2}$. Indeed, let $\mu>0$ be a sufficiently small constant given in Proposition 3.3. We divide $[0, T]$ into subintervals $J_{k}, k=1, \ldots, L$ in such a way that

$$
\left\|u_{\lambda}\right\|_{M^{\sigma}\left(J_{k}\right)} \leq \mu \text {. }
$$


The number of possible subinterval must satisfy

$$
L \sim\left(\frac{\lambda^{\gamma_{c}+\frac{\sigma(4-d) \gamma_{c}}{2(d-5+4 \sigma)}}}{\mu}\right)^{\frac{d-5+4 \sigma}{\sigma}} \sim \lambda^{\frac{\gamma_{c}(d-5+(8-d) \sigma)}{\sigma}} .
$$

We next apply Proposition 3.3 on each of the subintervals $J_{k}$ to have

$$
\sup _{[0, T]}\left\|I u_{\lambda}(t)\right\|_{L_{x}^{2}}^{2} \lesssim \sup _{[0, T]} E\left(I u_{\lambda}(t)\right) \leq E\left(I u_{\lambda}(0)\right)+C\left(E\left(I u_{\lambda}(0)\right)\right) N^{-(2-\gamma+\delta)} L .
$$

Since $E\left(I u_{\lambda}(0)\right) \leq \frac{1}{4}$, we need

$$
N^{-(2-\gamma+\delta)} L \ll \frac{1}{4}
$$

in order to guarantee (4.19) holds. Combining (4.5), (4.20) and (4.21), we need to choose $N$ depending on $\left\|u_{0}\right\|_{H_{x}^{\gamma}}$ such that

$$
N^{\frac{\gamma_{\mathrm{c}}(2-\gamma)(d-5+(8-d) \sigma)}{\left(\gamma-\gamma_{\mathrm{c}}\right) \sigma}-(2-\gamma+\delta)} \ll 1 .
$$

This is possible whenever $\gamma$ is such that

or

$$
\frac{\gamma_{\mathrm{c}}(2-\gamma)(d-5+(8-d) \sigma)}{\left(\gamma-\gamma_{\mathrm{c}}\right) \sigma}<2-\gamma+\delta,
$$

$$
\gamma_{\mathrm{c}}(2-\gamma)(d-5+(8-d) \sigma)<(2-\gamma+\delta)\left(\gamma-\gamma_{\mathrm{c}}\right) \sigma .
$$

Since $\delta<\min \left\{2 \gamma-3-\frac{\gamma_{c}}{2}, \gamma+\nu-4,(\nu-1) \gamma-2-(\nu-2) \gamma_{c}\right\}$, we have $\gamma>\gamma(d, \nu, \sigma)$, where $\gamma(d, \nu, \sigma)$ is the (larger if there are two) root of the equation

$$
\gamma_{\mathrm{c}}(2-\gamma)(d-5+(8-d) \sigma)=\min \left\{\gamma-1-\frac{\gamma_{\mathrm{c}}}{2}, \nu-2,(\nu-2)\left(\gamma-\gamma_{\mathrm{c}}\right)\right\}\left(\gamma-\gamma_{\mathrm{c}}\right) \sigma .
$$

This completes the bootstrap argument and (4.12) follows. Thus, (4.19) holds for all $T \in \mathbb{R}$.

We now estimate $\|u(T)\|_{H_{x}^{\gamma}}$. To do so, we use the conservation of mass, the scaling (1.2) and (2.9) to have

$$
\begin{aligned}
\|u(T)\|_{H_{x}^{\gamma}} & \lesssim\|u(T)\|_{L_{x}^{2}}+\|u(T)\|_{\dot{H}_{x}^{\gamma}} \\
& \lesssim\left\|u_{0}\right\|_{L_{x}^{2}}+\lambda^{\gamma-\gamma_{c}}\left\|u_{\lambda}\left(\lambda^{4} T\right)\right\|_{\dot{H}_{x}^{\gamma}} \\
& \lesssim\left\|u_{0}\right\|_{L_{x}^{2}}+\lambda^{\gamma-\gamma_{c}}\left\|I u_{\lambda}\left(\lambda^{4} T\right)\right\|_{H_{x}^{2}} \\
& \lesssim\left\|u_{0}\right\|_{L_{x}^{2}}+\lambda^{\gamma-\gamma_{c}}\left(\left\|u_{\lambda}\left(\lambda^{4} T\right)\right\|_{L_{x}^{2}}+\left\|I u_{\lambda}\left(\lambda^{4} T\right)\right\|_{\dot{H}_{x}^{2}}\right) .
\end{aligned}
$$

Using (4.19), we get for all $T \in \mathbb{R}$,

$$
\|u(T)\|_{H_{x}^{\gamma}} \lesssim\left\|u_{0}\right\|_{L_{x}^{2}}+\lambda^{\gamma-\gamma_{c}}\left(\lambda_{\mathrm{c}}^{\gamma}\left\|u_{0}\right\|_{L_{x}^{2}}+1\right) \leq C\left(\left\|u_{0}\right\|_{H_{x}^{\gamma}}\right) .
$$

Here we use (4.5) with the fact that $N$ is chosen sufficiently large depending only on $\left\|u_{0}\right\|_{H_{x}^{\gamma}}$. This proves (4.2) and the proof of Proposition 4.1 is complete.

Scattering. We firstly show that the global Morawetz estimate (4.1) can be upgraded to the global Strichartz estimate

$$
\|u\|_{S^{\gamma}(\mathbb{R})}:=\sup _{(p, q) \in B}\left\|\langle\nabla\rangle^{\gamma} u\right\|_{L_{t}^{p}\left(\mathbb{R}, L_{x}^{q}\right)} \leq C\left(\left\|u_{0}\right\|_{H_{x}^{\gamma}}\right) .
$$


Here we refer to Section 2 for the definition of $(p, q) \in B$. Let $u$ be a global solution to (NL4S) with initial data $u_{0} \in H^{\gamma}\left(\mathbb{R}^{d}\right)$ for $5 \leq d \leq 11$ and $\gamma(d, \nu)<\gamma<2$. Using the uniform bound (4.1), we can decompose $\mathbb{R}$ into a finite number of disjoint intervals $J_{k}=\left[t_{k}, t_{k+1}\right], k=1, \ldots, L$ so that

$$
\|u\|_{M^{\sigma}\left(J_{k}\right)} \leq \delta
$$

for a small constant $\delta>0$ to be chosen later. By Strichartz estimates (2.4) and (2.5), we have

$$
\|u\|_{S^{\gamma}\left(J_{k}\right)} \lesssim\left\|\langle\nabla\rangle^{\gamma} u\left(t_{k}\right)\right\|_{L_{x}^{2}}+\|F(u)\|_{L_{t}^{2}\left(J_{k}, L_{x}^{\frac{2 d}{d+4}}\right)}+\left\||\nabla|^{\gamma-1} F(u)\right\|_{L_{t}^{2}\left(J_{k}, L_{x}^{\frac{2 d}{d+2}}\right)} .
$$

We estimate for some $\varepsilon>0$,

$$
\begin{aligned}
& \left\||\nabla|^{\gamma-1} F(u)\right\|_{L_{t}^{2}\left(J_{k}, L_{x}^{\frac{2 d}{d+2}}\right)} \lesssim\left\||\nabla|^{\gamma-1} u\right\|_{L_{t}^{2+\varepsilon}\left(J_{k}, L_{x}^{\left.\frac{2 d(2+\varepsilon)}{(d-2)(2+\varepsilon)-8}\right)}\right.}\left\|F^{\prime}(u)\right\|_{L_{t}^{\frac{2(2+\varepsilon)}{\varepsilon}}\left(J_{k}, L_{x}^{\frac{d(2+\varepsilon)}{2 \varepsilon+8}}\right)} \\
& \lesssim\left\||\nabla|^{\gamma} u\right\|_{L_{t}^{2+\varepsilon}\left(J_{k}, L_{x}^{\frac{2 d(2+\varepsilon)}{d(2+\varepsilon)-8}}\right)}\|u\|_{L_{t}^{\nu-1}}^{\frac{2(\nu-1)(2+\varepsilon)}{\varepsilon}}\left(J_{k}, L_{x}^{\frac{d(\nu-1)(2+\varepsilon)}{2 \varepsilon+8}}\right) \\
& \lesssim\|u\|_{S^{\gamma}\left(J_{k}\right)}\|u\|_{L_{t}^{\frac{2(\nu-1)(2+\varepsilon)}{\varepsilon}}\left(J_{k}, L_{x} \frac{d(\nu-1)(2+\varepsilon)}{2 \varepsilon+8}\right)}^{\nu} .
\end{aligned}
$$

Similarly,

$$
\begin{aligned}
& \|\mid F(u)\|_{L_{t}^{2}\left(J_{k}, L_{x}^{\frac{2 d}{d+4}}\right)} \lesssim\|u\|_{L_{t}^{2+\varepsilon}\left(J_{k}, L_{x}^{\left.\frac{2 d(2+\varepsilon)}{d(2+\varepsilon)-8}\right)}\right.}\left\|F^{\prime}(u)\right\|_{L_{t}^{\frac{2(2+\varepsilon)}{\varepsilon}}\left(J_{k}, L_{x}^{\frac{d(2+\varepsilon)}{2 \varepsilon+8}}\right)} \\
& \lesssim\|u\|_{L_{t}^{2+\varepsilon}\left(J_{k}, L_{x}^{\left.\frac{2 d(2+\varepsilon)}{d(2+\varepsilon)-8}\right)}\right.}\|u\|_{L_{t}^{\frac{2(\nu-1)(2+\varepsilon)}{\varepsilon}}\left(J_{k}, L_{x} \frac{d(\nu-1)(2+\varepsilon)}{2 \varepsilon+8}\right)} \\
& \lesssim\|u\|_{S^{\gamma}\left(J_{k}\right)}\|u\|_{L_{t}^{\frac{2(\nu-1)(2+\varepsilon)}{\varepsilon}}\left(J_{k}, L_{x} \frac{d(\nu-1)(2+\varepsilon)}{2 \varepsilon+8}\right)} .
\end{aligned}
$$

We now need the following result.

Lemma 4.2. Let $d \geq 5,0<\sigma \leq \gamma<2$ be such that $\frac{(d-4) \sigma}{d-5+4 \sigma}<\gamma$ and $\frac{8}{d}<\nu-1<\frac{8}{d-2 \gamma}$. Then there exists $\varepsilon>0$ small such that for any time interval $J$,

$$
\|u\|_{L_{t}^{\frac{2(\nu-1)(2+\varepsilon)}{\varepsilon}}\left(J, L_{x} \frac{d(\nu-1)(2+\varepsilon)}{2 \varepsilon+8}\right)}^{\nu(1)} \lesssim\|u\|_{M^{\sigma}(J)}^{\frac{\varepsilon(d-5+4 \sigma)}{2(2+\varepsilon) \sigma}}\|u\|_{L_{t}^{\infty}\left(J, L_{x}^{2}\right)}^{\alpha(\varepsilon)}\|u\|_{L_{t}^{\infty}\left(J, \dot{H}_{x}^{\gamma}\right)}^{\beta(\varepsilon)}
$$

where

$\alpha(\varepsilon):=\left(1-\frac{d}{2 \gamma}\right)(\nu-1)+\frac{16 \sigma+\varepsilon((d+4) \sigma-\gamma(d-5+4 \sigma))}{2 \gamma \sigma(2+\varepsilon)}, \quad \beta(\varepsilon):=\frac{d}{\gamma}\left(\frac{\nu-1}{2}-\frac{16+\varepsilon(d+4)}{2 d(2+\varepsilon)}\right)$.

Proof. We firstly use Hölder's inequality to have

$$
\|u\|_{L_{t}^{\frac{2(\nu-1)(2+\varepsilon)}{\varepsilon}}\left(J, L_{x}\right.}^{\left.\frac{d(\nu-1)(2+\varepsilon)}{2 \varepsilon+8}\right)} \leq\|u\|_{M^{\sigma}(J)}^{\theta_{1}}\|u\|_{L_{t}^{\infty}\left(J, L_{x}^{q}\right)}^{1-\theta_{1}},
$$

provided that

$$
\frac{1-\theta_{1}}{q}=\frac{4(2 \varepsilon+8) \sigma-d \varepsilon(d-5+2 \sigma)}{4 d \sigma(\nu-1)(2+\varepsilon)} \quad \text { and } \quad \theta_{1}:=\frac{\varepsilon(d-5+4 \sigma)}{2(\nu-1)(2+\varepsilon) \sigma} .
$$

Similarly,

$$
\|u\|_{L_{t}^{\infty}\left(J, L_{x}^{q}\right)} \lesssim\|u\|_{L_{t}^{\infty}\left(J, L_{x}^{2}\right)}^{\theta_{2}}\|u\|_{L_{t}^{\infty}\left(J, L_{x}^{\frac{2 d}{d-2 \gamma}}\right)}^{1-\theta_{2}} \lesssim\|u\|_{L_{t}^{\infty}\left(J, L_{x}^{2}\right)}^{\theta_{2}}\|u\|_{L_{t}^{\infty}\left(J, \dot{H}_{x}^{\gamma}\right)}^{1-\theta_{2}},
$$

provided that

$$
\frac{1}{q}=\frac{\theta_{2}}{2}+\frac{\left(1-\theta_{2}\right)(d-2 \gamma)}{2 d}
$$


Thus, by (4.29) and (4.30), a direct consequence gives

$$
\|u\|_{L_{t}^{\frac{2(\nu-1)(2+\varepsilon)}{\varepsilon}}\left(J, L_{x} \frac{d(\nu-1)(2+\varepsilon)}{2 \varepsilon+8}\right)}^{\nu-1} \lesssim\|u\|_{M^{\sigma}(J)}^{\frac{\varepsilon(d-5+4 \sigma)}{2(2+\varepsilon) \sigma}}\|u\|_{L_{t}^{\infty}\left(J, L_{x}^{2}\right)}^{\alpha(\varepsilon)}\|u\|_{L_{t}^{\infty}\left(J, \dot{H}_{x}^{\gamma}\right)}^{\beta(\varepsilon)},
$$

where

$$
\begin{aligned}
& \alpha(\varepsilon):=\theta_{2}\left(1-\theta_{1}\right)(\nu-1)=\left(1-\frac{d}{2 \gamma}\right)(\nu-1)+\frac{16 \sigma+\varepsilon((d+4) \sigma-\gamma(d-5+4 \sigma))}{2 \gamma \sigma(2+\varepsilon)}, \\
& \beta(\varepsilon):=\left(1-\theta_{2}\right)\left(1-\theta_{1}\right)(\nu-1)=\frac{d}{\gamma}\left(\frac{\nu-1}{2}-\frac{16+\varepsilon(d+4)}{2 d(2+\varepsilon)}\right) .
\end{aligned}
$$

In order to perform the above estimates, we need $\alpha(\varepsilon)>0$ and $\beta(\varepsilon)>0$. We note that $\varepsilon \mapsto \alpha(\varepsilon)$ and $\varepsilon \mapsto \beta(\varepsilon)$ are decreasing functions provided that $\gamma>\frac{(d-4) \sigma}{d-5+4 \sigma}$. Moreover, since

$$
\alpha(\varepsilon) \rightarrow\left(1-\frac{d}{2 \gamma}\right)(\nu-1)+\frac{4}{\gamma}, \quad \beta(\varepsilon) \rightarrow \frac{d}{\gamma}\left(\frac{\nu-1}{2}-\frac{4}{d}\right) \text { as } \varepsilon \rightarrow 0 .
$$

As $\frac{8}{d}<\nu-1<\frac{8}{d-2 \gamma}$, the two limits are positive. Thus by taking $\varepsilon>0$ small enough, we have $\alpha(\varepsilon)>0$ and $\beta(\varepsilon)>0$. The proof is complete.

Remark 4.3. It is easy to see that the function $\sigma \in(0, \gamma] \mapsto \frac{(d-4) \sigma}{d-5+4 \sigma}$ is increasing and attains its maximal value at $\sigma=\gamma$. In this case, the condition $\frac{(d-4) \sigma}{d-5+4 \sigma}<\gamma$ becomes $\gamma>\frac{1}{4}$ which is always satisfied in our consideration.

We now continue the proof of scattering property. By (4.25), (4.26), (4.27) and Lemma 4.2, we have

$$
\begin{aligned}
\|u\|_{S^{\gamma}\left(J_{k}\right)} & \lesssim\left\|\langle\nabla\rangle^{\gamma} u\left(t_{k}\right)\right\|_{L_{x}^{2}}+\|u\|_{S^{\gamma}\left(J_{k}\right)}\|u\|_{M^{\sigma}\left(J_{k}\right)}^{\frac{\varepsilon(d-5+4 \sigma)}{2(2+\varepsilon) \sigma}}\|u\|_{L_{t}^{\infty}\left(J_{k}, L_{x}^{2}\right)}^{\alpha(\varepsilon)}\|u\|_{L_{t}^{\infty}\left(J_{k}, \dot{H}_{x}^{\gamma}\right)}^{\beta(\varepsilon)} \\
& \lesssim\left\|\langle\nabla\rangle^{\gamma} u\left(t_{k}\right)\right\|_{L_{x}^{2}}+\|u\|_{S^{\gamma}\left(J_{k}\right)}\|u\|_{M^{\sigma}\left(J_{k}\right)}^{\frac{\varepsilon(d-5+4 \sigma)}{2(2+\varepsilon) \sigma}}\|u\|_{L_{t}^{\infty}\left(J_{k}, H_{x}^{\gamma}\right)}^{\alpha(\varepsilon)+\beta(\varepsilon)} .
\end{aligned}
$$

This shows that

$$
\|u\|_{S^{\gamma}\left(J_{k}\right)} \lesssim\left\|\langle\nabla\rangle^{\gamma} u\left(t_{k}\right)\right\|_{L_{x}^{2}}+\|u\|_{S^{\gamma}\left(J_{k}\right)} \delta^{\frac{\varepsilon(d-5+4 \sigma)}{2(2+\varepsilon) \sigma}} C\left(\left\|u_{0}\right\|_{H_{x}^{\gamma}}\right) .
$$

By taking $\delta>0$ small enough, we get

$$
\|u\|_{S^{\gamma}\left(J_{k}\right)} \lesssim\left\|\langle\nabla\rangle^{\gamma} u\left(t_{k}\right)\right\|_{L_{x}^{2}} \leq C\left(\left\|u_{0}\right\|_{H_{x}^{\gamma}}\right)
$$

This proves (4.23).

We next use the global Strichartz bound (4.23) to prove the scattering property, i.e. there exist unique $u_{0}^{ \pm} \in H_{x}^{\gamma}$ such that

$$
\lim _{t \rightarrow \pm \infty}\left\|u(t)-e^{i t \Delta^{2}} u_{0}^{ \pm}\right\|_{H_{x}^{\gamma}}=0
$$

By the time reversal symmetry, it is enough to treat the positive time only. We will show that $e^{-i t \Delta^{2}} u(t)$ has limits in $H_{x}^{\gamma}$ as $t \rightarrow+\infty$. By Duhamel formula,

$$
e^{-i t \Delta^{2}} u(t)=u_{0}+i \int_{0}^{t} e^{-i s \Delta^{2}} F(u(s)) d s .
$$

For $0<t_{1}<t_{2}$, we have

$$
e^{-i t_{2} \Delta^{2}} u\left(t_{2}\right)-e^{-i t_{1} \Delta^{2}} u\left(t_{1}\right)=i \int_{t_{1}}^{t_{2}} e^{-i s \Delta^{2}} F(u(s)) d s .
$$


By Strichartz estimates (2.4), (2.5) and estimating as in (4.31),

$$
\begin{aligned}
\left\|e^{-i t_{2} \Delta^{2}} u\left(t_{2}\right)-e^{-i t_{1} \Delta^{2}} u\left(t_{1}\right)\right\|_{H_{x}^{\gamma}} & \lesssim\left\|i \int_{t_{1}}^{t_{2}} e^{-i s \Delta^{2}} F(u(s)) d s\right\|_{H_{x}^{\gamma}} \\
& \left.\lesssim\|F(u)\|_{L_{t}^{2}\left(\left[t_{1}, t_{2}\right], L_{x}^{\frac{2 d}{d+4}}\right)}+\left\||\nabla|^{\gamma-1} F(u)\right\|_{L_{t}^{2}\left(\left[t_{1}, t_{2}\right], L_{x}\right.}^{\frac{2 d}{d+2}}\right) \\
& \lesssim\|u\|_{S^{\gamma}\left(\left[t_{1}, t_{2}\right]\right)}\|u\|_{M^{\sigma}\left(\left[t_{1}, t_{2}\right]\right)}^{\frac{\varepsilon(d-5+4 \sigma)}{2(2+\varepsilon)}}\|u\|_{L_{t}^{\infty}\left(\left[t_{1}, t_{2}\right], H_{x}^{\gamma}\right)}^{\alpha(\varepsilon)+(\varepsilon)} \\
& \lesssim\|u\|_{S^{\gamma}\left(\left[t_{1}, t_{2}\right]\right)}^{1+\alpha(\varepsilon)}\|u\|_{M^{\sigma}\left(\left[t_{1}, t_{2}\right]\right)}^{\frac{\varepsilon(d-5+4 \sigma)}{2(2+\varepsilon)}} .
\end{aligned}
$$

This implies that $\left\|e^{-i t_{2} \Delta^{2}} u\left(t_{2}\right)-e^{-i t_{1} \Delta^{2}} u\left(t_{1}\right)\right\|_{H_{x}^{\gamma}} \rightarrow 0$ as $t_{1}, t_{2} \rightarrow+\infty$. Hence the limit

$$
u_{0}^{+}:=\lim _{t \rightarrow+\infty} e^{-i t \Delta^{2}} u(t)
$$

exists in $H_{x}^{\gamma}$. Moreover,

$$
u(t)-e^{i t \Delta^{2}} u_{0}^{+}=-i \int_{t}^{+\infty} e^{i(t-s) \Delta^{2}} F(u(s)) d s .
$$

A same argument as above shows that

$$
\left\|u(t)-e^{i t \Delta^{2}} u_{0}^{+}\right\|_{H_{x}^{\gamma}} \rightarrow 0
$$

as $t \rightarrow+\infty$. The proof is now complete.

\section{ACKNowledgments}

The author would like to express his deep thanks to his wife - Uyen Cong for her encouragement and support. He would like to thank his supervisor Prof. Jean-Marc Bouclet for the kind guidance and constant encouragement. He also would like to thank the reviewer for his/her helpful comments and suggestions.

\section{REFERENCES}

[BKS00] M. Ben-Artzi, H. Koch, J. C. Saut Disperion estimates for fourth-order Schrödinger equations, C.R.A.S., 330, Série 1, 87-92 (2000). 1, 7

[BCD11] H. Bahouri, J. Y. Chemin, R. Danchin, Fourier analysis and non-linear partial differential equations, A Series of Comprehensive Studies in Mathematis 343, Springer (2011). 7

[CW91] M. Christ, I. Weinstein, Dispersion of small amplitude solutions of the generalized Korteweg-de Vries equation, J. Funct. Anal. 100, No. 1, 87-109 (1991). 6

[CKSTT02] J. Colliander, M. Keel, G. Staffilani, H. Takaoka, T. Tao, Almost conservation laws and global rough solutions to a nonlinear Schrödinger equation, Math. Res. Lett. 9, 659-682 (2002). 2, 3

[CKSTT04] J. Colliander, M. Keel, G. Staffilani, H. Takaoka, T. Tao, Global existence and scattering for rough solutions of a nonlinear Schrödinger equation on $\mathbb{R}^{3}$, Comm. Pure Appl. Math. 57, 987-1014 (2004). 18

[DPST07] D. De Silva, N. Pavlovic, G. Staffilani, N. Tzirakis, Global well-posedness for the $L^{2}$-critical nonlinear Schrödinger equation in higher dimensions, Commun. Pure Appl. Anal. 6, No. 4, 1023-1041 (2007).

[Din1] V. D. Dinh, Well-posedness of nonlinear fractional Schrödinger and wave equations in Sobolev spaces, arXiv:1609.06181 (2016). 1, 2, 7

[Din2] V. D. Dinh, On well-posedness, regularity and ill-posedness for the nonlinear fourth-order Schrödinger equation, to appear in Bull. Belg. Math. Soc Simon Stevin, arXiv:1703.00891 (2018). 1, 2

[Din3] V. D. Dinh, Global existence for the defocusing mass-critical nonlinear fourth-order Schrödinger equation below the energy space, Unpublished, arXiv:1706:06517 (2017). 1, 2, 8

[Din4] V. D. Dinh, On the defocusing mass-critical nonlinear fourth-order Schrodinger equation below the energy space, Dyn. Partial Differ. Equ. 14, No. 3, 295-320 (2017). 1

[FIP02] G. Fibich, B. Ilan, G. Papanicolaou, Self-focusing with fourth order dispersion, SIAM J. Appl. Math. 62, No. 4, 1437-1462 (2002). 
[Guo10] C. Guo, Global existence of solutions for a fourth-order nonlinear Schrödinger equation in $n+1$ dimensions, Nonlinear Anal. 73, 555-563 (2010). 1, 2

[GC06] C. Guo, S. Cui, Global existence of solutions for a fourth-order Schrödinger equation, Appl. Math. Lett. 19, 706-711 (2006). 1

[HHW06] C. Hao, L. Hsiao, B. Wang, Well-posedness for the fourth-order Schrödinger equations, J. Math. Anal. Appl. 320, 246-265 (2006). 1

[HHW07] C. Hao, L. Hsiao, B. Wang, Well-posedness of the Cauchy problem for the fourth-order Schrödinger equations in high dimensions, J. Math. Anal. Appl. 328, 58-83 (2007). 1

[HJ05] Z. Huo, Y. Jia, The Cauchy problem for the fourth-order nonlinear Schrödinger equation related to the vortex filament, J. Differential Equations 214, 1-35 (2005). 1

[Kar96] V. I. Karpman, Stabilization of soliton instabilities by higher-order dispersion: Fourth order nonlinear Schrödinger-type equations, Phys. Rev. E 53 (2), 1336-1339 (1996). 1

[KS00] V. I. Karpman, A.G Shagalov, Stability of soliton described by nonlinear Schrödinger-type equations with higher-order dispersion, Phys. D 144, 194-210 (2000). 1

[KPV93] C. E. Kenig, G. Ponce, L. Vega, Well-posedness and scattering results for the gereralized Korteveg-de Vries equation via the contraction principle, Comm. Pure Appl. Math 46, 527-620 (1993). 6

[MXZ09] C. Miao, G. Xu, L. Zhao, Global well-posedness and scattering for the defocusing energy critical nonlinear Schrödinger equations of fourth-order in the radial case, J. Differ. Eqn. 246, 3715-3749 (2009). 1

[MXZ11] C. Miao, G. Xu, L. Zhao, Global well-posedness and scattering for the defocusing energy critical nonlinear Schrödinger equations of fourth-order in dimensions $d \geq 9$, J. Differ. Eqn. 251, 3381-3402 (2011). 1

[MWZ15] C. Miao, H. Wu, J. Zhang, Scattering theory below energy for the cubic fourth-order Schrödinger equation, Math. Nachr. 288, No. 7, 798-823 (2015). 1, 2, 3, 4, 8, 18

[MZ07] C. Miao, B. Zhang, Global well-posedness of the Cauchy problem for nonlinear Schrödinger-type equations, Discrete Contin. Dyn. Syst. 17, No. 1, 181-200 (2007). 1, 7

[Pau1] B. Pausader, Global well-posedness for energy critical fourth-order Schrödinger equations in the radial case, Dynamics of PDE 4, No. 3, 197-225 (2007). 1, 7

[Pau2] B. Pausader, The cubic fourth-order Schrödinger equation, J. Funct. Anal. 256, 2473-2517 (2009). 1, 4, 7,

[PS10] B. Pausader, S. Shao, The mass-critical fourth-order Schrödinger equation in higher dimensions, J. Hyper. Differential Equations 7, No. 4, 651-705 (2010). 1

[Tao06] T. Tao, Nonlinear dispersive equations: local and global analysis, CBMS Regional Conference Series in Mathematis 106, AMS (2006). 7

[VZ09] M. Visan, X. Zhang, Global well-posedness and scattering for a class of nonlinear Schrödinger equations below the energy space, Differ. Integral Eqn. 22, 99-124 (2009). 2, 3, 4, 8

[Vis06] M. Visan, The focusing energy-critical nonlinear Schrödinger equation in dimensions five and higher, $\mathrm{PhD}$ Thesis, UCLA (2006). 6

(V. D. Dinh) Institut de Mathématiques de Toulouse UMR5219, Université Toulouse CNRS, 31062

Toulouse Cedex 9, France

E-mail address: dinhvan.duong@math.univ-toulouse.fr 